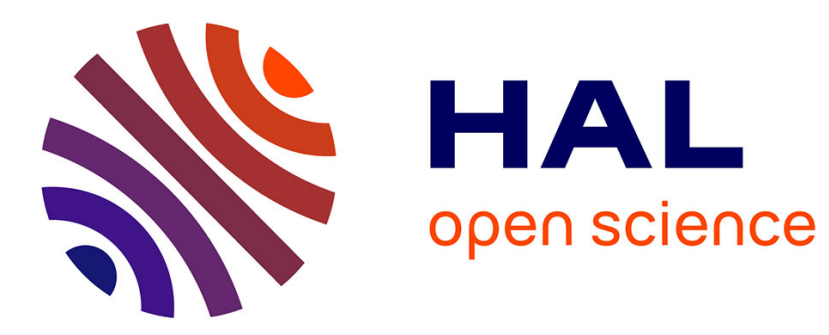

\title{
Generalization of Heisenberg Hamiltonians to non half-filled bands: a magneto-angular effective Hamiltonian for boron clusters
}

\author{
A. Pellegatti, F. Marinelli, M. Roche, Daniel Maynau, Jean-Paul Malrieu
}

\section{To cite this version:}

A. Pellegatti, F. Marinelli, M. Roche, Daniel Maynau, Jean-Paul Malrieu. Generalization of Heisenberg Hamiltonians to non half-filled bands : a magneto-angular effective Hamiltonian for boron clusters. Journal de Physique, 1987, 48 (1), pp.29-43. 10.1051/jphys:0198700480102900 . jpa-00210421

\section{HAL Id: jpa-00210421 https://hal.science/jpa-00210421}

Submitted on 1 Jan 1987

HAL is a multi-disciplinary open access archive for the deposit and dissemination of scientific research documents, whether they are published or not. The documents may come from teaching and research institutions in France or abroad, or from public or private research centers.
L'archive ouverte pluridisciplinaire HAL, est destinée au dépôt et à la diffusion de documents scientifiques de niveau recherche, publiés ou non, émanant des établissements d'enseignement et de recherche français ou étrangers, des laboratoires publics ou privés. 
Classification

Physics Abstracts

$31.10-31.20 \mathrm{D}-31.20 \mathrm{R}-75.10$

\title{
Generalization of Heisenberg Hamiltonians to non half-filled bands : a magneto-angular effective Hamiltonian for boron clusters
}

\author{
A. Pellegatti, F. Marinelli, M. Roche $(*)$, D. Maynau $\left(^{+}\right)$and J. P. Malrieu $\left(^{+}\right)$ \\ Centre de Thermodynamique et de Microcalorimétrie, (U.P. 7461 du C.N.R.S.), 26, rue du $141^{\mathrm{e}}$ R.I.A., \\ 13003 Marseille, France \\ $\left(^{+}\right)$Laboratoire de Physique Quantique, (U.A. 505 du C.N.R.S.), Université Paul Sabatier, \\ 118, route de Narbonne, 31062 Toulouse Cedex, France
}

(Reçu le 26 mars 1986, accepté le 16 septembre 1986)

\begin{abstract}
Résumé. - On propose un hamiltonien effectif pour l'étude des états neutres d'agrégats de Bore, en généralisant les hamiltoniens de Heisenberg au cas d'une bande remplie au $1 / 6^{\mathrm{e}}$. L'espace modèle est porté par les produits des fonctions fondamentales ${ }^{2} \mathrm{P}\left(\mathrm{s}^{2} \mathrm{p}\right)$ des atomes. L'hamiltonien effectif implique, outre des termes d'échange de spin de type Heisenberg, des opérateurs d'échange du moment angulaire et des opérateurs changeant à la fois le spin et le moment angulaire des atomes. La logique de cet hamiltonien effectif est obtenue par développement au $3^{\mathrm{e}}$ ordre de la théorie des perturbations quasi dégénérées. En pratique l'hamiltonien est obtenu directement par les équations de Bloch à partir de calculs précis des fonctions d'onde de $\mathrm{B}_{2}$. La transférabilité de cet hamiltonien effectif a été testée sur le système $B_{3}$ linéaire ; la partie basse du spectre est correctement reproduite. Ce modèle sera utilisé pour l'étude du ferromagnétisme possible de chaînes linéaires de Bore. L'article discute le rôle de l'hybridation et souligne la portée de cette approche, qui étend la rationalisation par Anderson des hamiltoniens de Heisenberg à des systèmes autres que les bandes $1 / 2$ remplies.
\end{abstract}

Abstract. - An effective Hamiltonian has been proposed for the study of the neutral states of boron clusters, i.e. for a 1/6th filled band. This generalization of Heisenberg Hamiltonians rests upon the definition of a model space spanned by products of ground state ${ }^{2} \mathrm{P}\left(\mathrm{s}^{2} \mathrm{p}\right)$ atomic wave-functions. The resulting effective Hamiltonian involves, besides Heisenberg-type effective spin exchange terms, angular-momentum exchange operators and spin-andangular-momentum exchange operators. The logic of this effective Hamiltonian has been derived through $3 \mathrm{~d}$ order quasi-degenerate perturbation theory. In practice the effective interactions are obtained from accurate MO-CI large

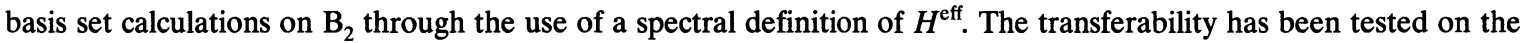
linear $\mathrm{B}_{3}$ system, by comparing the $H^{\text {eff }}$ predictions with accurate MO-CI results. The transferability proves to be satisfactory for the lower part of the spectrum. The model will be used for the treatment of the magnetic character of (possibly high spin) $\mathbf{B}_{n}$ linear chains. The role of hybridization processes has been discussed and the possible extension of this approach, derived from Anderson's rationalization of Heisenberg Hamiltonians, has been outlined.

\section{Introduction.}

The theoretical foundations of Heisenberg Hamiltonians [1] are known to be based on the theory of effective Hamiltonians and on the use of quasi-degenerate perturbation theory [2]. These effective Hamiltonians are easy to derive for systems involving one electron in one Atomic Orbital (AO) on each centre [2], and (although less evidently [3]) for systems

$\left({ }^{*}\right)$ Permanent address : Département de Chimie, Université de Provence, Place Victor Hugo, 13331 Marseille Cedex 3, France. involving $\mathrm{p}$ electrons in $\mathrm{p}$ AO's per centre. In both cases the systems involve a half-filled-band (the other bands being full or empty). Besides their typical applications for Mott insulators and magnetic problems in coordination chemistry, the Heisenberg Hamiltonians have proved to be surprinsingly efficient tools for problems where the electronics delocalization is far from being small, namely in organic chemistry for the study of conjugated molecules [4] and even for alkali atom clusters or solids [5].

The basic idea of the present work is to attempt an extension of this approach to non-half filled bands, for 
instance to $1 / 6$-filled bands, as will occur in $s^{2} p$ atoms (B or Al clusters). The motivation and the strategy are developed in section 2 ; the choice of the model space, spanned by the Valence Bond (VB) determinants built from ground state ${ }^{2} \mathrm{P}\left(\mathrm{s}^{2} \mathrm{p}\right)$ atomic wave-functions, is explained and discussed. The ferromagnetic character of the $\mathrm{B}_{2}$ ground state suggests a possible ferromagnetic ordering of the linear $\mathrm{B}_{n}$ chains.

Section 3 derives the formal structure of the effective Hamiltonian from the application of the quasi-degenerate perturbation theory (to third order) to the $B_{2}$ problem. It is shown that, besides angular dependant repulsion terms and other first order terms, the effective Hamiltonian presents a large variety of operators, of second-and third-order origin. These operators act on local spins only, angular momenta only or on both spin and angular momenta, and imply 2 AO's only (of either the same or orthogonal subsystem) or 4 AO's. The effective Hamiltonian for this problem is thus a magneto-angular effective Hamiltonian since it acts on both the local (i.e. atomic) spin and angular momenta. Its formal structure is developed in second-quantization formalism. From accurate molecular orbital configuration interaction (MO-CI) and orthogonal V.B. calculations on $\mathrm{B}_{2}$ numerical effective Hamiltonians are proposed, refering to a direct (non-perturbative) definition of the effective Hamiltonian, analogous to those of Bloch [6] and Des Cloiseaux [7].

In a fourth section the transferability of these effective Hamiltonians to heavier systems is tested on the linear $\mathrm{B}_{3}$ problem. For this molecule accurate $\mathrm{MO}-\mathrm{CI}$ calculations have been performed assessing the exact state ordering. It appears that in this system all the lowest neutral states essentially keep the $s^{2} p$ character of the atoms. The effective Hamiltonian, extracted from the relevant lowest eigenstates of $B_{2}$, gives surprisingly accurate energies for all the lowest states of $\mathrm{B}_{3}$ of various spin multiplicities and symmetries. This implicit treatment of the hybridization problem is discussed and a further generalization of the approach to the simultaneous treatment of different bands is suggested. Applications to longer linear chains is planned for the near future.

\section{Situation of the problem.}

2.1 HALF-FILLED BANDS : THE HEISENBERG HAMILTONIANS. - For a system involving $n$ electrons and $n$ active AO's, the procedure to obtain Heisenberg Hamiltonians consists first in the definition of a model space; this model space is spanned by the Valence Bond single determinants which occupy each of the $n$ active AO's of the system once and only once. Then all the determinants of the model space have the same space part ; they only differ by the spin distribution, and the model space is composed of $C_{n}^{n / 2}$ determinants. These determinants are all neutral in the sense of V.B. theory, and they are the determinants of lowest energy in the V.B. matrix (at least in non-polar well-defined problems). The second step of the procedure consists in applying the QDPT [8] to this model space, i.e. to construct perturbatively the effective energies and effective coupling of the neutral determinants under the influence of the ionic determinants. For a twoelectron, two-AO ( $\mathrm{a}$ and b), two-centre system, Anderson shows that the effective exchange between the two neutral determinants $|\mathrm{a} \overline{\mathrm{b}}|$ and $|\overline{\mathrm{a}} \mathrm{b}|$ is

$$
\left\langle|\mathrm{a} \overline{\mathrm{b}}|\left|H^{\mathrm{eff}}\right||\overline{\mathrm{a}} \mathrm{b}|\right\rangle=-K_{\mathrm{ab}}+F_{\mathrm{ab}}^{2} / \Delta E
$$

where $K_{\mathrm{ab}}$ is the direct bicentric exchange, $F_{\mathrm{ab}}$ the hopping integral between the two centres and $\Delta E$ is the "transition" energy from the neutral to the ionic $|a \bar{a}|$ or $|b \bar{b}|$ determinants. The Heisenberg Hamiltonian treats the electronic delocalization (i.e. the mixture between neutral and ionic determinants) as effective spin operators between the neutral determinants.

This straight forward approach is not directly generalizable to the general half-filled band problems ( $p$ electrons in $p$ AO's per centre), if one uses the wellknown Bloch [6] or Des Cloizeaux [7] definitions of the effective Hamiltonian, as shown by Maynau et al. [3] recently ; new definitions of the effective Hamiltonian are to be proposed to obtain a good transferability of the effective couplings from the two-centre problem to larger systems. Nevertheless the general foundations of the Heisenberg Hamiltonians for half-filled band problems are clear.

The Heisenberg Hamiltonians have recently received new applications, far from the typical field of Mott insulators. They have been applied with surprising success to the $\pi$-systems of conjugated molecules in organic chemistry [4]. A precise MO-CI calculation on ethylene makes possible the accurate extraction of two operators (a spin-dependant $\sigma$ potential $+\pi$ repulsion and an effective exchange) which are functions of the interatomic distances. This Heisenberg Hamiltonian predicts very accurately the geometries, isomerization energies, rotational barriers, lowest excited states energies and conformations of conjugated molecules involving up to 10 carbon atoms [4].

A similar attempt has been performed on an isoelectronic problem, namely the alkali atom clusters. A Heisenberg Hamiltonian has been extracted from accurate $\mathrm{MO}-\mathrm{CI}$ potential curves of $\mathrm{Na}_{2}$; it proves to give realistic energies of small clusters and the cohesive energy of the bulk [5].

The application of $a b$ initio extracted efficient Heisenberg-type Hamiltonian for the study of metal clusters may appear as a tempting challenge in view of the difficulty of usual $a b$ initio MO-CI techniques for the treatment of such versatile systems, presenting certainly several nearly degenerate optimal conformations with possibly different spin multiplicities. 
2.2. NON HALF-FILLED BAND : TOWARDS NEW TYPES OF EFFECTIVE HAMILTONIANS. - The basic purpose of the present paper is to establish the form of an effective Hamiltonian, inspired by the strategy leading to the Heisenberg Hamiltonians but dealing with non half-filled bands. The fundamental idea is that, as in Anderson's legitimization of the Heisenberg Hamiltonian, one may :

i) choose as model space the set of neutral determinants with maximum spread of the electrons in the concerned band,

ii) apply the quasi degenerate perturbation theory, or an alternative variational formalism [9], to build the effective Hamiltonian spanned by this model space, to treat the effect of ionic configurations on the neutral subspace.

As a model problem we consider a one-sixth (1/6)filled band, i.e. a cluster of $\mathrm{B}$ (or $\mathrm{Al}$ ) atoms in their $\mathrm{s}^{2} \mathrm{p}\left({ }^{2} \mathrm{P}\right)$ ground state. The $\mathrm{s}$ band is supposed to be filled up, and the hybridization will be considered as a perturbation only. We choose the model space $S_{0}$ as built of all the determinants in which each atom $\mathrm{A}$ bears one (and only one) p-electron in a $\mathrm{p}_{x}, \mathrm{p}_{y}$ or $\mathrm{p}_{z}$ AO. The projector $P_{0}$ on the model space is written as :

$$
P_{0}=\sum_{K \in \mathrm{S}_{0}}|K\rangle\langle K|
$$

where

$$
|K\rangle=\mathcal{A} \prod_{\mathrm{A}}\left[\left(\mathrm{s}_{\mathrm{A}}^{2}\right) \mathrm{p}_{\mathrm{A}, i_{\mathrm{A}}}\right], \quad i_{\mathrm{A}}=x, y, z .
$$

Then it is clear that the model space determinants do not have the same space-part; for each atom one has two degrees of freedom namely the orientation $x, y$ or $z$ of the occupied $\mathrm{AO}$ and its spin. The size of the effective matrix for a $2 n$-atom problem is $3^{2 n} \mathrm{C}_{2 n}^{n}$, larger than for a typical Heisenberg Hamiltonian, but tremendously smaller than the full V.B.-matrix size.

The logic of the effective Hamiltonian is of course different from that of a Heisenberg Hamiltonian since it deals with both the local spin and angular momentum. One may expect that the effective operators will be both bicentric spin exchange operators and l-exchange operators, as will be confirmed in the next section. The logical structure of the effective Hamiltonian has to be established from analytic perturbative developments using the quasi degenerate perturbation theory on a two centre problem in the spirit of the AndersonBrandow approach to Heisenberg Hamiltonians.

Two types of problems may arise along this path :

- non-convergence of the quasi degenerate perturbation expression, especially at short distances;

- mixing with hybridized structures in which the $\mathrm{sp}^{2}$ excited atomic configuration becomes dominant.

These configurations may appear as intruder states in the QDPT expansion, questionning the validity of the choise of the model space. This problem will be briefly discussed in the present paper but the perturbative difficulties will be avoided by a spectral definition of the effective Hamiltonian. The generalization of this approach to the simultaneous treatment of several bands is out of the scope of the present work.

2.3 InTEREST IN B CLUSTERS. - At least at long distances, the reduction of the model space to the determinants built from the $\mathrm{s}^{2} \mathrm{p}\left({ }^{2} \mathrm{P}\right)$ ground state of the atoms is valid ; the lowest $\mathrm{sp}^{2}\left({ }^{2} \mathrm{P}\right)$ atomic state is $3.5 \mathrm{eV}$ above the ground state, and the corresponding $\mathrm{sp}^{2}\left({ }^{2} \mathrm{D}\right)$ is $6 \mathrm{eV}$ above the ground state. The ground state of $B_{2}$ is of ${ }^{3} \Sigma_{g}^{-}$symmetry, is essentially built from $\mathrm{A}\left(\mathrm{s}^{2} \mathrm{p}_{x}\right) \times \mathrm{B}\left(\mathrm{s}^{2} \mathrm{p}_{y}\right)$ atomic configurations, and is ferromagnetic :
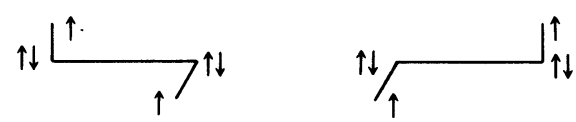

This point is interesting since it suggest that :

i) the $s^{2} p$ configuration may dominate the ground state of at least linear B clusters.

ii) the linear B chain may be a high spin compound since it may be viewed as dominated by a spin-angular

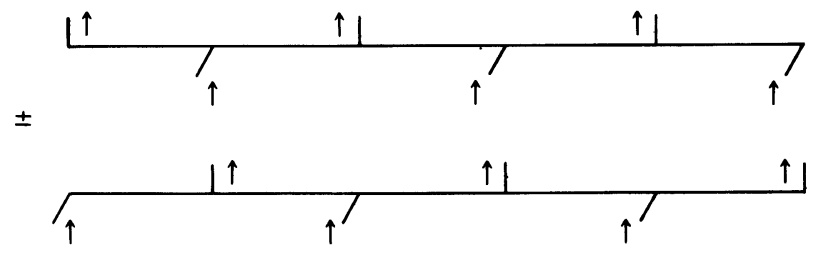

arrangement i.e. presenting a molecular magnet. Besides the formal problem treated here, the present work may then be of practical interest.

\section{Structure of the $B_{2}$ effective Hamiltonian.}

3.1 LOWER PART OF THE SPECTRUM FROM A MO-CI APPROACH. - The number of papers related to be $\mathrm{B}_{2}$ molecule is rather limited. Among those concerning the order of the low-lying states, let us note first the calculations of Padgett and Griffing [10] at the SCF level and of Bender and Davidson [11] at the CI level. In the last, the CI was not sufficiently extended so that the ${ }^{5} \Sigma_{u}^{-}$is assigned to the ground state in both papers, instead of the true ${ }^{3} \Sigma_{\mathrm{g}}^{-}$state [12]. At the same time, Bader, Henneker and Cade [13] focused their attention on the charge distributions and the chemical binding. More recently Dupuis and Liu [14] performed a more extended CI calculation and found a good agreement between their results and Douglas and Herzberg's experimental interpretation of the symmetry of the ground state $\left({ }^{3} \Sigma_{\mathrm{g}}^{-}\right)$and of the first ${ }^{3} \Sigma_{\mathrm{u}}^{-} \Leftrightarrow{ }^{3} \Sigma_{\mathrm{g}}^{-}$ 
transition. The ${ }^{5} \Sigma_{u}^{-}$state was found 0.006 a.u. higher than the ground state. Becke [15] using a numerical Hartree-Fock-Slater method studied only the ground state ${ }^{3} \Sigma_{\mathrm{g}}^{-}$. He found a dissociation energy of $3.8 \mathrm{eV}$, slightly larger than the experimental value

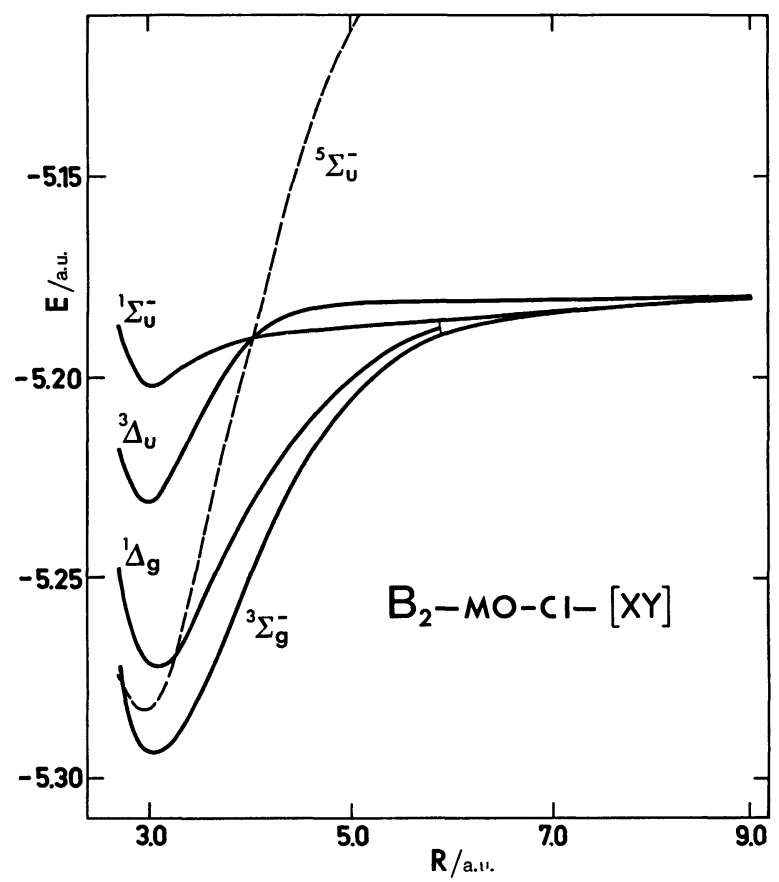

Fig. 1. - MO-CI $[x y]$-symmetry potential curves of $\mathrm{B}_{2}$.

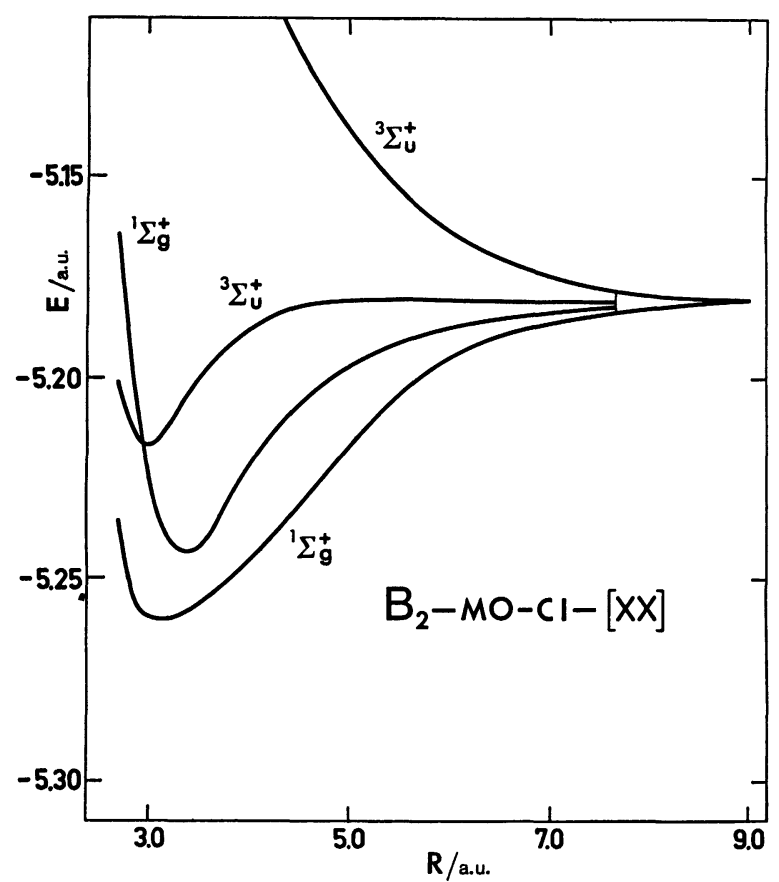

Fig. 2. - MO-CI $[x x, y y, z z]$-symmetry potential curves of $\mathrm{B}_{2} . \Delta$ states, degenerate with those of the $[x y]$-symmetry have been removed from the figure.
$(3.1 \pm 0.2 \mathrm{eV}[16])$. All these calculations found an interatomic distance close to the experimental value 3.005 a.u. [17]. The most accurate results are those of Dupuis and Liu but unfortunately they only concern the $\mathrm{X}^{3} \Sigma_{\mathrm{g}}^{-}, a^{5} \Sigma_{\mathrm{u}}^{-}$and the two first ${ }^{3} \Sigma_{\mathrm{u}}^{-}$states.

As will be seen in next sections, a series of potential curves of $B_{2}$ are required for the construction of the desired effective Hamiltonians. By the way, it is also interesting to test the validity of our MO-CI calculations for this simple case and in particular concerning the small difference separating the ${ }^{3} \Sigma_{\mathrm{g}}^{-}$and ${ }^{5} \Sigma_{\mathrm{u}}^{-}$states. At the SCF level we used the Pshondo program [18], an extansion of Hondo [19]. From the basis evaluated for the $B$ atom [20] we extracted a triple- $\zeta$ s (contracted [3, $1,1])$, a double- $\zeta \mathrm{p}$ (contracted $[3,1])$ and one single- $\zeta \mathrm{d}$ basis set. The $d$-exponent parameter was chosen equal to 0.5 . Correlation effects have been introducted by means of the CIPSI algorithm [21]. In this method, a multiconfigurational zero-order wave function is first obtained from a variational configuration interaction (CI) calculation in a restricted subspace built up from the most important determinants for the states being studied; then this wave function is perturbed up to second order in energy.

The ${ }^{3} \Sigma_{\mathrm{g}}^{-}$state is clearly found to be the ground state. The spectroscopic characteristics $\quad\left(r_{\mathrm{e}}=3.05\right.$ a.u., $\left.D_{\mathrm{c}}=3.10 \mathrm{eV}\right)$ are in good agreement with experiment $\left(r_{\mathrm{c}}=3.005\right.$ a.u., $\left.D_{\mathrm{e}}=3.10 \mathrm{eV}\right)$. As also predicted by Dupuis and Liu, the ${ }^{5} \Sigma_{\mathrm{u}}^{-}$state is obtained at 0.01 a.u. above the ground state, with a slightly smaller equilib-

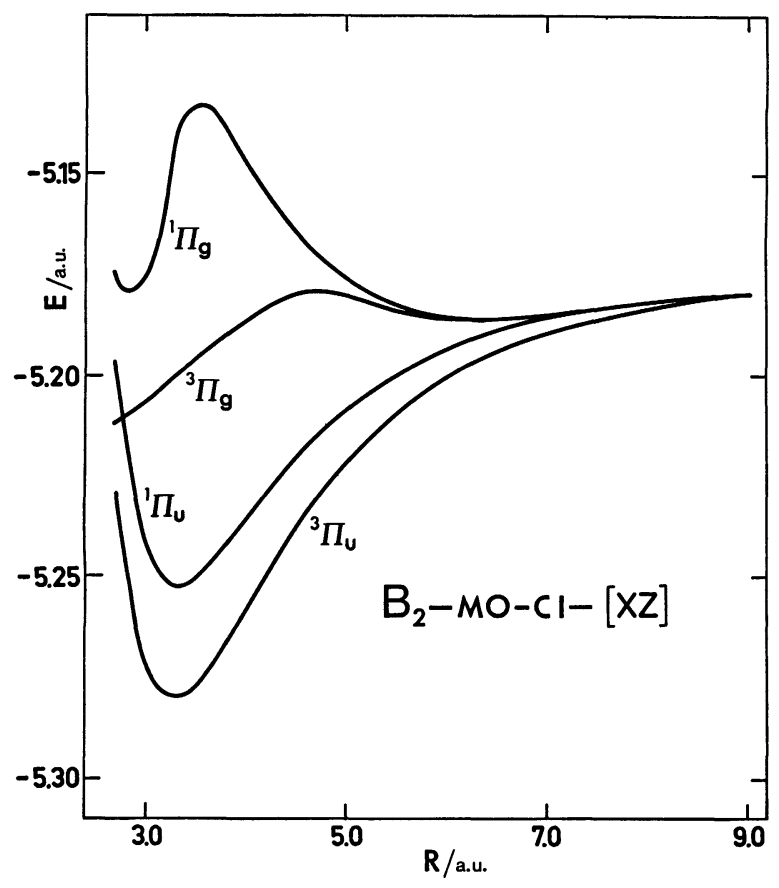

Fig. 3. - MO-CI $[x z]$-symmetry potential curves of $\mathrm{B}_{2}$. 
rium distance $\left(r_{\mathrm{e}}=3.00\right.$ a.u. $)$. One must immediately notice that this ${ }^{5} \Sigma_{u}^{-}$state is necessarily spanned by $B$ $\mathrm{sp}^{2}\left({ }^{2} \mathrm{D}\right) \times \mathrm{B} \mathrm{s}^{2} \mathrm{p}\left({ }^{2} \mathrm{P}\right)$ V.B. structures, and it is necessarily outside of our model space (cf. Eq. (1)).

The relevant potential curves of $B_{2}$ are pictured in figures 1-3. Curves corresponding to various irreductible representations of the $D_{\infty h}$ point group have been gathered into three reducible representations, which may be reached from the use of $\mathrm{p}_{x} \mathrm{p}_{z}$ and $\mathrm{p}_{z}$ AO's $(z$ being the internuclear axis) ; this presentation is especially convenient for the forthcoming construction of the effective Hamiltonians. The figures only report the potential curves of the states having significant components in the model space (with the exception of the above mentioned ${ }^{5} \Sigma_{u}^{-}$state, which appears as a dotted line). The concerned states are always the lower ones of their symmetry, with three exceptions only : the highest in each symmetry. Other states, of redundant (spin or space) symmetry and/or of irrelevant V.B. character were found between the two reported ${ }^{3} \Sigma_{\mathrm{u}}^{+},{ }^{3} \Delta \mathrm{u}$ and ${ }^{1} \Sigma_{\mathrm{u}}^{-},{ }^{3} \Pi_{\mathrm{g}}$ and ${ }^{1} \Pi_{\mathrm{g}}$ states. In particular, one notices irregular shapes of the ${ }^{3} \Pi_{\mathrm{g}}$ and ${ }^{1} \Pi_{\mathrm{g}}$ potential curves, which result from an avoided crossing with upper configurations. Since the corresponding eigenstates kept, at short interatomic distances, a non-negligible (although non-dominant) component in the model V.B. space, we decided to use these states in the definition of the «target» stable subspace defining $H^{\text {eff }}$. This may be called an adiabatic definition of the target space S (see below for a more detailed discussion).

\subsection{Definition of the EFFective Hamiltonian.}

3.2.1 Model space, size and symmetry partitioning. According to the basic choice of our treatment, the model space for $B_{2}$ reduces to the neutral $A\left(s^{2} p\right) \times B\left(s^{2} p\right)$ V.B. determinants (cf. Eq. (1)).

$$
\begin{aligned}
P_{0}=\sum_{\substack{i, j \\
x, y, z}} \|\left(\mathrm{s}_{\mathrm{A}}^{2}\right) \mathrm{p}_{\mathrm{A}, i}\left(\mathrm{~s}_{\mathrm{B}}^{2}\right) \mathrm{p}_{\mathrm{B}, j}|\rangle \times & \\
& \times\langle|\left(\mathrm{s}_{\mathrm{A}}^{2}\right) \mathrm{p}_{\mathrm{A}, i}\left(\mathrm{~s}_{\mathrm{B}}^{2}\right) \mathrm{p}_{\mathrm{B}, j} \|
\end{aligned}
$$

where $\mathrm{p}_{\mathrm{A}, i}$ is a spin orbital $\mathrm{P} x, y$ or $z$ on the atom $\mathrm{A}$. The model space splits into $S_{z}= \pm 1, S_{z}=0$ independent subspaces, the last one being the largest one and containing all the relevant information. Equations (1) and (2) implicity assume the use of orthogonal valence AO's. This may be achieved by a preliminary $S^{-1 / 2}$ transformation, but, as noticed elsewhere $[4,5]$, the precise specification of this basis is not necessary. This orthogonality problem, connected with transferability to larger systems, will be discussed in the subsequent section. This model space may be split into three different symmetries $\quad\left[x_{\mathrm{a}} x_{\mathrm{b}}, y_{\mathrm{a}} y_{\mathrm{b}}, z_{\mathrm{a}} z_{\mathrm{b}}\right]$, $\left[x_{\mathrm{a}} y_{\mathrm{b}}, y_{\mathrm{a}} x_{\mathrm{b}}\right]$ and $\left[x_{\mathrm{a}} z_{\mathrm{b}}, z_{\mathrm{a}} x_{\mathrm{b}}\right]$ (and its degenerate $\left[y_{\mathrm{a}} z_{\mathrm{b}}, z_{\mathrm{a}} y_{\mathrm{b}}\right]$ counterpart) where the atoms are denoted $a$ and $b$ for convenience. The size of the effective matrices will be 6,4 and 4 respectively for $S_{z}=0$, and $3,2,2$, for $S_{z}= \pm 1$. The $\mathrm{u}, \mathrm{g}$ symmetry will be exploited later on.

Once the model space is defined the effective Hamiltonian may be obtained by two different routes :

- the perturbative approach, using quasidegenerate perturbation theory in one of its versions ;

- the direct use of the Bloch or Des Cloiseaux equations, since in that precise problem, almost exact energies and wave functions are available from the MO-CI preliminary calculations. The latter solution is equivalent to an infinite order summation of the perturbative expansion, and is by far more precise, but the low-order perturbative approach gives the main structure of the effective Hamiltonian and the physical origin of the effective interactions.

3.2.2 Structure of the effective Hamiltonian from third order perturbative expansion. - Some details about the general structure were given in previous papers [3-5]. Let us recall that the perturbation operator is defined by :

$$
H=H_{0}+V
$$

with

$$
H_{0}=\sum_{\mathrm{K} \in \mathrm{S}_{0}} E_{\mathrm{K}}^{0}|\mathrm{~K}\rangle\left\langle\mathrm{K}\left|+\sum_{\alpha \notin \mathrm{S}_{0}} E_{\alpha}^{0}\right| \alpha\right\rangle\langle\alpha|
$$

where $\mathrm{S}_{0}$ is the model space. In an Epstein-Nesbet definition [22] of $H_{0}$, the $V$ operator is purely extradiagonal. Up to second order the effective Hamiltonian matrix elements take the form :

$$
\begin{aligned}
\left\langle\mathrm{I}\left|H^{\text {eff }}(2)\right| \mathrm{J}\right\rangle=\langle\mathrm{I}|H| \mathrm{J}\rangle & \\
& -\sum_{\alpha \notin \mathrm{S}_{0}} \frac{\langle\mathrm{I}|H| \alpha\rangle\langle\alpha|H| \mathrm{J}\rangle}{E_{\alpha}^{0}-E_{\mathrm{J}}^{0}}
\end{aligned}
$$

and to third order

$$
\begin{aligned}
\langle\mathrm{I}| H^{\text {eff (3) }|\mathrm{J}\rangle} & =\langle\mathrm{I}| H^{\text {eff (2) }|\mathrm{J}\rangle} \\
+ & \sum_{\alpha, \beta \notin \mathrm{s}_{0}} \frac{\langle\mathrm{I}|H| \alpha\rangle\langle\alpha|H| \beta\rangle\langle\beta|H| \mathrm{J}\rangle}{\left(E_{\alpha}^{0}-E_{\mathrm{J}}^{0}\right)\left(E_{\beta}^{0}-E_{\mathrm{J}}^{0}\right)} \\
& -\sum_{\alpha \notin \mathrm{s}_{0}} \sum_{\mathrm{L} \in \mathrm{S}_{0}} \frac{\langle\mathrm{I}|H| \alpha\rangle\langle\alpha|H| \mathrm{L}\rangle\langle\mathrm{L}|H| \mathrm{J}\rangle}{\left(E_{\alpha}^{0}-E_{\mathrm{J}}^{0}\right)^{2}}
\end{aligned}
$$

where $\alpha, \beta$ may be ionic or hybridized neutral determinants.

Since the ionic determinants are known to play the dominant role in the interpretation of the effective antiferromagnetic coupling in half-filled bands, we shall concentrate our attention on their effect in our problem. If one restricts the V.B. minimal set of determin- 
ants to those which keep $\mathrm{s}^{2}$ electron pairs on each atom, the V.B. matrix may be schematically pictured as in figure 4 , for the $[x x, y y, z z]$ symmetry, which is the largest and most complex one. The zeroth order energies of the neutral determinants are repulsive, but to a larger extent for $z_{\mathrm{a}} z_{\mathrm{b}}$ spatial parts $\left(R_{z z}>R_{x x}\right)$; the same differences appear among ionic states. The largest extradiagonal elements are hopping integrals $F$ coupling the neutral $x_{\mathrm{a}} \bar{x}_{\mathrm{b}}$ (resp. $y_{\mathrm{a}} \bar{y}_{\mathrm{b}}$ or $z_{\mathrm{a}} \bar{z}_{\mathrm{b}}$ ) and ionic $x_{\mathrm{a}} \bar{x}_{\mathrm{a}}$ (resp. $y_{\mathrm{a}} \bar{y}_{\mathrm{a}}$ or $z_{\mathrm{a}} \bar{z}_{\mathrm{a}}$ ) determinants. One should also notice the occurrence of non-negligible monocentric exchange integrals $K$ between ionic determinants. The $h_{x y}\left(\right.$ and $h_{x z}$ ) integrals represent electrostatic interactions between the atomic $x y$ (or $x z$ ) quadrupoles ; the other integrals involve bicentric overlap distributions and are much smaller.

To first order, the different symmetries involve 6 parameters

$$
\begin{aligned}
& R_{1}=R_{x x}=R_{y y} ; R_{1}^{\prime}=R_{z z} \\
& R_{2}=R_{x y} \\
& R_{3}=R_{x z}=R_{y z} \\
& h=\left(x_{\mathrm{a}} y_{\mathrm{a}}, x_{\mathrm{b}} y_{\mathrm{b}}\right) \\
& h^{\prime}=\left(x_{\mathrm{a}} z_{\mathrm{a}}, x_{\mathrm{b}} z_{\mathrm{b}}\right)=\left(y_{\mathrm{a}} z_{\mathrm{a}}, y_{\mathrm{b}} z_{\mathrm{b}}\right) .
\end{aligned}
$$

The identity of the $\Delta_{x y}$ and $\Delta_{x^{2}-y^{2}}$ solutions at first order leads to the following relation

$$
R_{1}=R_{2}+2 h .
$$

Starting from the neutral $x_{\mathrm{a}} \bar{x}_{\mathrm{b}}$ determinant one may go to an ionic $x_{\mathrm{a}} \bar{x}_{\mathrm{a}}$ or $x_{\mathrm{b}} \bar{x}_{\mathrm{b}}$ determinant. From it one may either move back to the $x_{\mathrm{a}} \bar{x}_{\mathrm{b}}$ original determinant (and obtain a diagonal second order correction) or reach the $x_{\mathrm{b}} \bar{x}_{\mathrm{a}}$ determinant ; the effective coupling is then an interatomic exchange, shown in the following scheme where ${ }^{*}$ and $O$ stand for $\alpha$ and $\beta$ spins respectively.

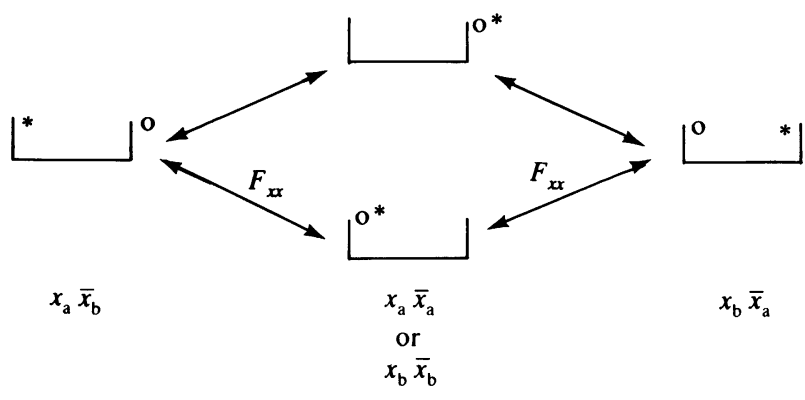

This back-and-forth second order process in the $\pi_{x}$ subsystem repeats the well-known Anderson's mechanism of antiferromagnetic coupling. One may write diagonal $-g_{x x}\left|x_{\mathrm{a}} \bar{x}_{\mathrm{b}}\right\rangle\left\langle x_{\mathrm{a}} \bar{x}_{\mathrm{b}}\right|$ and off-diagonal $-g_{x x}\left|x_{\mathrm{a}} \bar{x}_{\mathrm{b}}\right\rangle\left\langle x_{\mathrm{b}} \bar{x}_{\mathrm{a}}\right|$ operators, with $g_{1}=g_{x x}=$ $2 F_{x x}^{2} / \Delta E_{x x}$, which give the amplitude of the negative effective exchange integral in the $\pi_{x}$ system. Analogous contributions occur in the $\pi_{y}\left(g_{y y}=g_{1}\right)$ and $\sigma\left(g_{z z}=g_{1}^{\prime}\right)$ systems.

A second type of coupling occurs at third order, pictured as follows

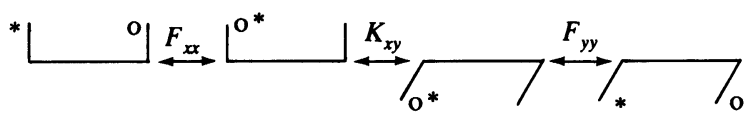

which couples $x_{\mathrm{a}} \bar{x}_{\mathrm{b}}$ and $y_{\mathrm{a}} \bar{y}_{\mathrm{b}}$ through

$$
\varepsilon_{x y}\left|x_{\mathrm{a}} \bar{x}_{\mathrm{b}}\right\rangle\left\langle y_{\mathrm{a}} \bar{y}_{\mathrm{b}}\left|=2 \frac{F_{x x} K_{x y} F_{y y}}{\Delta E_{x x}+\Delta E_{y y}}\right| x_{\mathrm{a}} \bar{x}_{\mathrm{b}}\right\rangle\left\langle y_{\mathrm{a}} \bar{y}_{\mathrm{b}}\right|
$$

(the factor 2 reflects the possible travel through

\begin{tabular}{|c|c|c|c|c|c|c|c|c|c|c|c|}
\hline$x_{a} \bar{x}_{b}$ & $\mathrm{x}_{\mathrm{b}} \bar{x}_{\mathrm{a}}$ & $y_{a^{y_{b}}}^{\bar{b}}$ & $y_{b} \bar{y}_{a}$ & $z_{a^{2} \bar{z}_{b}}$ & $z_{b} \bar{z}_{a}$ & $x_{a} \overline{a_{a}}$ & $x_{b} \bar{x}_{b}$ & $y_{a} \bar{y}_{a}$ & $y_{b} \bar{y}_{b}$ & $z_{a} \bar{z}_{a}$ & $z_{b} \bar{z}_{b}$ \\
\hline $\mathbf{R}_{\mathbf{x x}}$ & $R_{x x}$ & $\begin{array}{l}h_{x y} \\
R_{y y}\end{array}$ & $\begin{array}{l}h_{x y} \\
R_{y y}\end{array}$ & $\begin{array}{l}h_{x z} \\
h_{y z} \\
R_{z z}\end{array}$ & $\begin{array}{l}h_{x z} \\
h_{y z} \\
R_{z z}\end{array}$ & $\begin{array}{l}F_{x x} \\
F_{x x}\end{array}$ & $\begin{array}{l}F_{x x} \\
F_{x x}\end{array}$ & $\begin{array}{l}F_{y y} \\
F_{y y}\end{array}$ & $\begin{array}{l}F_{y y} \\
F_{y y}\end{array}$ & $\begin{array}{l}F_{z z} \\
F_{z z}\end{array}$ & $\begin{array}{l}F_{2 z} \\
F_{2 z}\end{array}$ \\
\hline & & & & & & $R_{x x}+\Delta E_{x x}$ & $R_{x x}+\Delta E_{x x}$ & $\begin{array}{r}K_{x y} \\
R_{y y}+\Delta E_{y y}\end{array}$ & $\begin{array}{r}K_{x y} \\
y_{y y}+\Delta E_{y y}\end{array}$ & $\begin{array}{l}K_{x z} \\
K_{y z} \\
z_{z z}+\Delta E_{z 2}\end{array}$ & $\begin{array}{l}K_{x z} \\
K_{y z} \\
z_{z z}+\Delta E_{z}\end{array}$ \\
\hline
\end{tabular}
$\mathrm{A}^{+} \mathrm{A}^{-}$and $\mathrm{A}^{-} \mathrm{B}^{+}$structures). This operator changes the angular part of the wave-function, and therefore cannot enter a Heisenberg Hamiltonian. Analogous operators occur between $x$ and $z$ or $y$ and $z$ spatial parts, with a different amplitude $\varepsilon_{x z}=\varepsilon_{y z}$. Table I summarizes the expected structure of the effective

Fig. 4. - Structure of the V.B. matrix spanned by the non hybridized, $S_{z}=0$, determinants in the $[x x, y y, z z]$-symmetry. Bicentric exchange integrals are not taken into account. Identities between matrix elements, resulting from the degeneracy of $\pi$ orbitals are explicitly given in table I. 


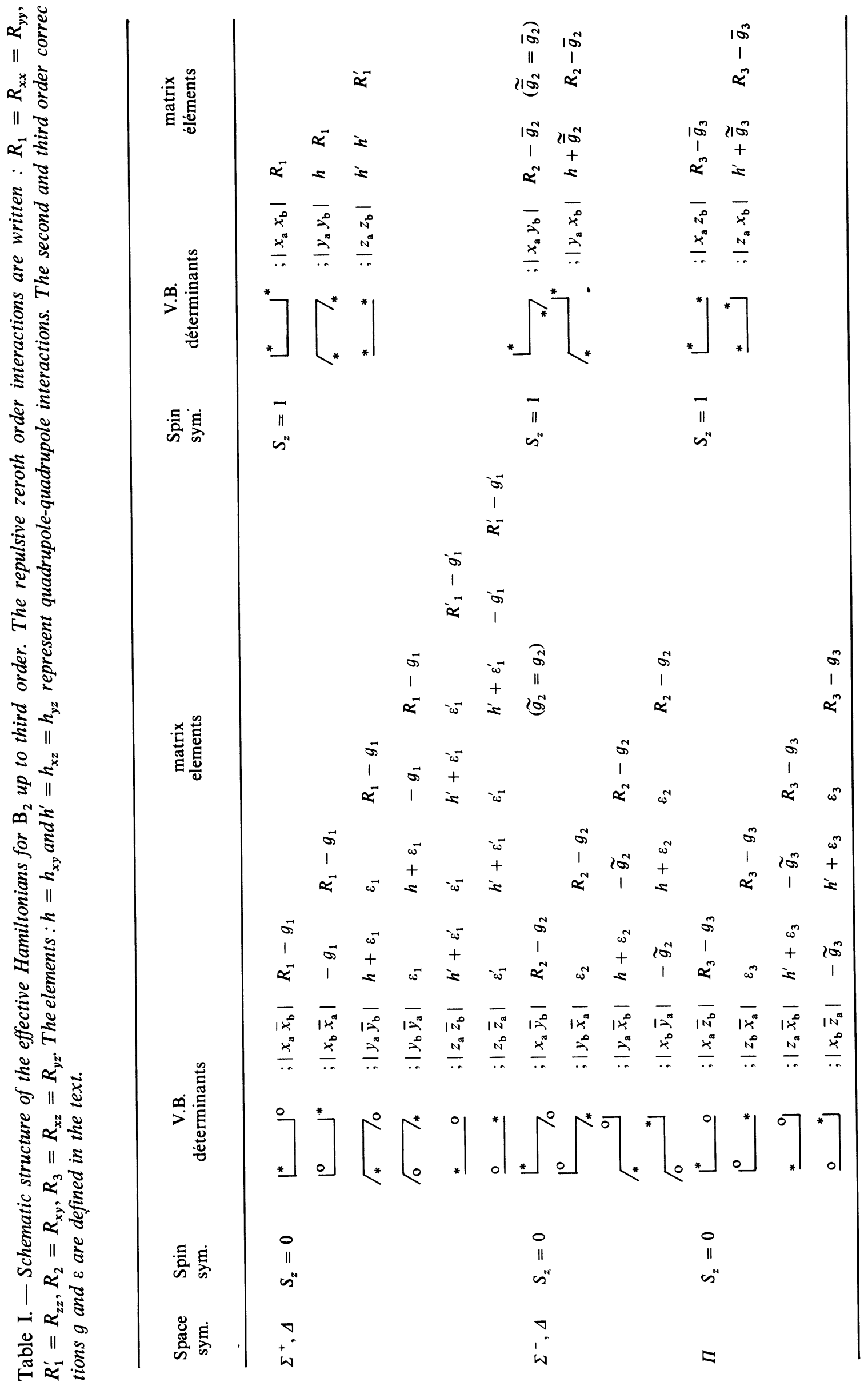


Hamiltonian in the $[x x, y y, z z]$ symmetry $\left(S_{z}=0\right.$ and 1).

In the $[x y]$ symmetry, analogous processes take place leading to second order diagonal and off-diagonal corrections,

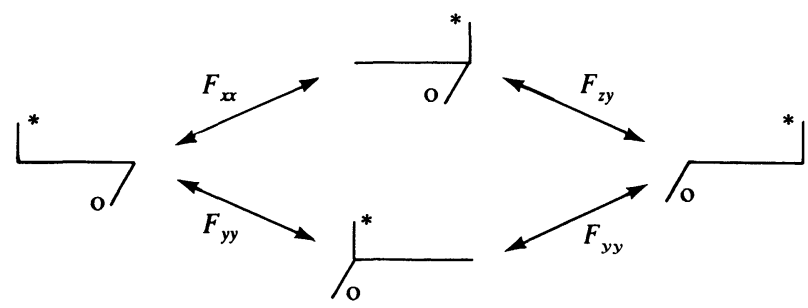

with for example the corresponding off-diagonal operator

$$
g_{x y}\left|x_{\mathrm{a}} \bar{y}_{\mathrm{b}}\right\rangle\left\langle x_{\mathrm{b}} \bar{y}_{\mathrm{a}}\left|=2 \frac{F_{x x} F_{y y}}{\Delta E_{x y}}\right| x_{\mathrm{a}} \bar{y}_{\mathrm{b}}\right\rangle\left\langle x_{\mathrm{b}} \bar{y}_{\mathrm{a}}\right|
$$

where one may notice that $\Delta E_{x y}=\Delta E_{x x}-2 K_{x y}$, since one does not go through an ionic determinant with 2 electrons in the same AO, as occurred in the Anderson typical problem and in the $[x x, y y, z z]$ symmetry.

A third order contribution also appears through processes such as

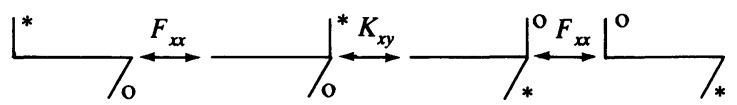

leading to non-Heisenberg operators

$$
\varepsilon_{2}\left|x_{\mathrm{a}} \bar{y}_{\mathrm{b}}\right\rangle\left\langle y_{\mathrm{b}} \bar{x}_{\mathrm{a}}\left|=2 \frac{F_{x x}^{2} K_{x y}}{\Delta E_{x y}^{2}}\right| x_{\mathrm{a}} \bar{y}_{\mathrm{b}}\right\rangle\left\langle y_{\mathrm{b}} \bar{x}_{\mathrm{a}}\right| .
$$

Table I gives the structure of the third order effective Hamiltonian in the $[x y]$ symmetry for $S_{z}=0$ and $S_{z}=1$. The identity of the $S_{z}=0$ and $S_{z}=1$ triplet eigenvalues requires that

$$
\bar{g}_{2}=g_{2}+\varepsilon_{2} .
$$

This relation is easily understood from the difference in the energy denominators

$$
\Delta E\left(S_{z}=1\right) \simeq \Delta E\left(S_{z}=0\right)-K
$$

so that

$$
\begin{aligned}
\bar{g}_{2} & =g_{2}+\frac{2 K F^{2}}{\Delta E^{2}}+\cdots \\
& =g_{2}+\varepsilon_{2} .
\end{aligned}
$$

Similar derivations are posible for the $[x z]$ (and $[y z]$ ) symmetry and are also shown in table I. However one should notice that :

i) the diagonal second order corrections are

$$
-\left(\frac{F_{x x}^{2}}{\Delta E}+\frac{F_{z z}^{2}}{\Delta E}\right)=-g_{3}
$$

while the off-diagonal second-order correction obtained from the operator

$$
-\tilde{g}_{3}\left|x_{\mathrm{a}} \bar{z}_{\mathrm{b}}\right\rangle\left\langle x_{\mathrm{b}} \bar{z}_{\mathrm{a}}\left|=-2 \frac{F_{x x} F_{z z}}{\Delta E}\right| x_{\mathrm{a}} \bar{z}_{\mathrm{b}}\right\rangle\left\langle x_{\mathrm{b}} \bar{z}_{\mathrm{a}}\right|
$$

is of different amplitude than the diagonal correction and

ii) the $F_{z z}$ hopping integral is positive, resulting in apparent changes in sign, also active in the $\varepsilon_{3}$ third order terms.

The identity of the ${ }^{3}\left(S_{z}=0\right)$ and ${ }^{3}\left(S_{z}=1\right)$ solutions for the $[x z]$ symmetry leads to the following relations

$$
\begin{aligned}
& \bar{g}_{3}=g_{3}+\varepsilon_{3} \\
& \tilde{\bar{g}}_{3}=\tilde{g}_{3}+\varepsilon_{3}
\end{aligned}
$$

analogous to equation (3).

3.2.3 Physical typology of the effective operators. One may summarize the various types of operators according to several criteria :

- diagonal/non-diagonal character ;

- order of perturbation concerned ;

- number of AO's involved ;

- action on the local spin and angular momenta.

On the diagonal one first has purely $\left(l_{\mathrm{a}}, l_{\mathrm{b}}\right)$ dependent terms $R$ which are independent of the spin. One also has $-g$ stabilization second order contributions. The terms

$$
-g_{1}\left|x_{\mathrm{a}} \bar{x}_{\mathrm{b}}\right\rangle\left\langle x_{\mathrm{a}} \bar{x}_{\mathrm{b}}\left|, \quad-g_{1}^{\prime}\right| z_{\mathrm{a}} \bar{z}_{\mathrm{b}}\right\rangle\left\langle z_{\mathrm{a}} \bar{z}_{\mathrm{b}}\right|
$$

only involve two AO's of the same subsystem, and appear when these two AO's bear electrons of different spins.

The $-g_{2}\left|x_{\mathrm{a}} \bar{y}_{\mathrm{b}}\right\rangle\left\langle x_{\mathrm{a}} \bar{y}_{\mathrm{b}}\left|, \quad-g_{3}\right| x_{\mathrm{a}} \bar{z}_{\mathrm{b}}\right\rangle\left\langle x_{\mathrm{a}} \bar{z}_{\mathrm{b}}\right|$ terms imply two AO's belonging to two orthogonal subsystems.

The

$$
-\bar{g}_{2}\left|x_{\mathrm{a}} y_{\mathrm{b}}\right\rangle\left\langle x_{\mathrm{a}} y_{\mathrm{b}}\left|, \quad-\bar{g}_{3}\right| x_{\mathrm{a}} z_{\mathrm{b}}\right\rangle\left\langle x_{\mathrm{a}} z_{\mathrm{b}}\right|
$$

operators appear in the $S_{z}=1$ (or -1 ) subspaces. They involve two AO's of two orthogonal subsystems bearing parallel spins.

Off the diagonal one finds typical second order spin exchange integrals involving only two AO's of the same subsystem, as in all Heisenberg Hamiltonians

$$
-g_{1}\left|x_{\mathrm{a}} \bar{x}_{\mathrm{b}}\right\rangle\left\langle x_{\mathrm{b}} \bar{x}_{\mathrm{a}}\right|
$$

and analogous operators for $y \bar{y}\left(-g_{1}\right)$ and $z \bar{z}\left(-g_{1}^{\prime}\right)$ determinants.

Other second order operators of the type

$$
-\tilde{g}_{2}\left|x_{\mathrm{a}} \bar{y}_{\mathrm{b}}\right\rangle\left\langle x_{\mathrm{b}} \bar{y}_{\mathrm{a}}\left|, \quad-\tilde{g}_{3}\right| x_{\mathrm{a}} \bar{z}_{\mathrm{b}}\right\rangle\left\langle x_{\mathrm{b}} \bar{z}_{\mathrm{a}}\right|
$$

change both the spin of the atom and its angular 
Table II. - Characters of the various off-diagonal effective operators.

\begin{tabular}{|c|c|c|c|c|}
\hline \multirow{2}{*}{$\begin{array}{l}H^{\text {eff }} \text { matrix } \\
\text { element }\end{array}$} & \multicolumn{2}{|c|}{ action on } & \multirow{2}{*}{$\begin{array}{c}\text { no. of } \\
\text { A.O.'s involved }\end{array}$} & \multirow{2}{*}{$\begin{array}{l}\text { perturb. } \\
\text { order. }\end{array}$} \\
\hline & atom-spin & atom-1 & & \\
\hline$g_{1}, g_{1}^{\prime}$ & + & - & 2 & 2 \\
\hline$\tilde{g}_{2}, \tilde{g}_{3}$ & + & + & 4 & 2 \\
\hline$\left(h+\tilde{\bar{g}}_{2}\right),\left(h^{\prime}+\tilde{\bar{g}}_{3}\right)$ & - & + & 4 & $1+2$ \\
\hline$\varepsilon_{1}, \varepsilon_{1}^{\prime}$ & + & + & 4 & 3 \\
\hline$\varepsilon_{2}, \varepsilon_{3}$ & + & - & 2 & 3 \\
\hline $\begin{array}{l}\left(h+\varepsilon_{1}\right),\left(h^{\prime}+\varepsilon_{1}^{\prime}\right) \\
\left(h+\varepsilon^{\prime}\right)\left(h^{\prime}+\varepsilon^{\prime}\right)\end{array}$ & - & + & 4 & $1+3$ \\
\hline
\end{tabular}

momentum. These operators involve four AO's belonging to two orthogonal subsystems.

The second order off-diagonal

$$
\begin{aligned}
& \left(h+\check{\bar{g}}_{2}\right)\left|x_{\mathrm{a}} y_{\mathrm{b}}\right\rangle\left\langle y_{\mathrm{a}} x_{\mathrm{b}}\right|, \\
& \left(h^{\prime}+\overline{\bar{g}}_{3}\right)\left|x_{\mathrm{a}} z_{\mathrm{b}}\right\rangle\left\langle z_{\mathrm{a}} x_{\mathrm{b}}\right|,
\end{aligned}
$$

operators change the angular momenta of the atoms without changing their spins. One should notice that due to the identity of the $\pi_{x}$ and $\pi_{y}$ systems $\breve{g}_{2}=g_{2}$ and $\underline{\bar{g}}=\bar{g}_{2}$.

The third order operators

$$
\varepsilon_{2}\left|x_{\mathrm{a}} y_{\mathrm{b}}\right\rangle\left\langle y_{\mathrm{b}} x_{\mathrm{a}}\left|, \quad \varepsilon_{3}\right| x_{\mathrm{a}} z_{\mathrm{b}}\right\rangle\left\langle z_{\mathrm{b}} x_{\mathrm{a}}\right|
$$

change the spins of two spin-orbitals of different spins belonging to two orthogonal subsystems, without acting on the space part.

The third-order operators

$$
\begin{gathered}
\left(h+\varepsilon_{1}\right)\left|x_{\mathrm{a}} \bar{x}_{\mathrm{b}}\right\rangle\left\langle y_{\mathrm{a}} \bar{y}_{\mathrm{b}}\right|, \\
\left(h^{\prime}+\varepsilon_{1}^{\prime}\right)\left|x_{\mathrm{a}} \bar{x}_{\mathrm{b}}\right\rangle\left\langle z_{\mathrm{a}} \bar{z}_{\mathrm{b}}\right|, \\
\left(h+\varepsilon_{2}\right)\left|x_{\mathrm{a}} \bar{y}_{\mathrm{b}}\right\rangle\left\langle y_{\mathrm{a}} \bar{x}_{\mathrm{b}}\right|, \\
\left(h^{\prime}+\varepsilon_{3}\right)\left|x_{\mathrm{a}} \bar{z}_{\mathrm{b}}\right\rangle\left\langle z_{\mathrm{a}} \bar{x}_{\mathrm{b}}\right|
\end{gathered}
$$

change the angular momentum on the atom without changing the local spin; they involve four atomic orbitals belonging to two orthogonal subsystems.

Finally the third-order operators

$$
\varepsilon_{1}\left|x_{\mathrm{a}} \bar{x}_{\mathrm{b}}\right\rangle\left\langle y_{\mathrm{b}} \bar{y}_{\mathrm{a}}\left|, \quad \varepsilon_{1}^{\prime}\right| x_{\mathrm{a}} \bar{x}_{\mathrm{b}}\right\rangle\left\langle z_{\mathrm{b}} \bar{z}_{\mathrm{a}}\right|
$$

change both the angular and spin momenta on the atom, involving therefore four AO's on two orthogonal subsystems. This typology is clearly pictured in table II.

3.3 AB INITIO DERIVATION OF THE EFFECTIVE HAMILTONIANS FROM THE BLOCH EQUATION. Since the effective Hamiltonian satisfies the Bloch equation for $n$ roots

$$
H^{\mathrm{eff}}\left|P_{0} \psi_{i}\right\rangle=E_{i}\left|P_{0} \psi_{i}\right\rangle \quad i=1, \ldots, n
$$

with

$$
H\left|\psi_{i}\right\rangle=E_{i}\left|\psi_{i}\right\rangle
$$

a spectral definition of $H^{\text {eff }}$ is given by

$$
H^{\mathrm{eff}}=\sum_{i=1}^{n}\left|P_{0} \psi_{i}\right\rangle E_{i}\left\langle P_{0} \psi_{i}^{+}\right|
$$

where $P_{0} \psi_{i}^{\perp}$ is the biorthogonal vector associated with the projection $P_{0} \psi_{i}$ of the eigenstate $\psi_{i}$ of $H$ into $\mathrm{S}_{0}$.

If the exact spectrum of $H$ and its eigenvectors are known, a selection of $n$ eigenvalues and eigenvectors $\psi_{i}$ defines

$$
P_{\mathrm{s}}=\sum_{i=1}^{n}\left|\psi_{i}\right\rangle\left\langle\psi_{i}\right|
$$

the stable subspace, which we call « target space », and $H^{\text {eff }}$ is entirely defined by $P_{0}, P_{\mathrm{s}}$ and the exact eigenenergies, without any reference to a possibly divergent perturbation expansion. If one uses the wave operator $\Omega$, then :

$$
P_{\mathrm{s}}=\Omega P_{0} .
$$

Since for the $B_{2}$ molecule, accurate energies and wave functions are available from the MO-CI calculations, it is tempting to use equation (5) to obtain directly the $H^{\text {eff }}$ matrix, in a purely numerical form : in this section the target space is defined as spanned by the eigenvectors of the correct symmetry, lowest energy and having significant components on the model space. Another definition of the target space might pick up the eigenvectors having the largest components onto the model space. From symmetry considerations, the following correspondence between model subspaces and eigenstates is expected :

$[x x, y y, z z] S_{z}=0$ corresponds to one ${ }^{1} \Delta_{\mathrm{g}}$, two $\left({ }^{1} \Sigma_{\mathrm{g}}^{+}\right)$, one $\left({ }^{3} \Delta_{\mathrm{u}}\right)$, two $\left({ }^{3} \Sigma_{\mathrm{u}}^{+}\right)$eigenstates, $\left(S_{z}=1\right.$ generates the triplets : one $\left({ }^{3} \Delta_{\mathrm{u}}\right)$, two $\left.\left({ }^{3} \Sigma_{\mathrm{u}}^{+}\right)\right)$. 
[xy] $\quad S_{z}=0$ corresponds to one ${ }^{3} \Sigma_{\mathrm{g}}^{-}$, one ${ }^{1} \Delta_{\mathrm{g}}$, one ${ }^{1} \Sigma_{\mathrm{u}}^{-}$and one ${ }^{3} \Delta_{\mathrm{u}}$ states, ( $S_{z}=1$ generates the corresponding triplet states).

$[x z] \quad S_{z}=0$ corresponds to one ${ }^{3} \Pi_{\mathrm{u}}$, one ${ }^{1} \Pi_{\mathrm{u}}$, one ${ }^{3} \Pi_{\mathrm{g}}$ and one ${ }^{1} \Pi_{\mathrm{g}}$ states, $\left(S_{z}=1\right.$ generates the corresponding triplet states).

The $\Delta$ states obtained from $[x x, y y, z z]$ and $[x y]$ model subspaces are of course degenerate.

All the vectors which are the only ones of their symmetry in the concerned model subspace have their components in the model space entirely defined by symmetry. This means that the components of $P_{0} \psi_{i}$ are immediately obtained. For instance for ${ }^{3} \Sigma_{\mathrm{g}}^{-}$one necessarily has +--+ components on the $\left|x_{\mathrm{a}} \bar{y}_{\mathrm{b}}\right|$, $\left|y_{\mathrm{b}} \bar{x}_{\mathrm{a}}\right|,\left|y_{\mathrm{a}} \bar{x}_{\mathrm{b}}\right|$ and $\left|x_{\mathrm{b}} \bar{y}_{\mathrm{a}}\right|$ model determinants. The only exception concerns the pairs of ${ }^{1} \Sigma_{\mathrm{g}}^{+}$and ${ }^{3} \Sigma_{\mathrm{u}}^{+}$states belonging to the $[x x, y y, z z]$ symmetry. The degree of freedom concerns of course the ratio of $\left|x_{\mathrm{a}} \bar{x}_{\mathrm{b}}\right|\left(=\left|y_{\mathrm{a}} \bar{y}_{\mathrm{b}}\right|\right)$ over $\left|z_{\mathrm{a}} \bar{z}_{\mathrm{b}}\right|$ components.

In principle the projected eigenvectors of the same symmetry are not orthogonal

$$
\left\langle P_{0} \psi_{i} \mid P_{0} \psi_{j}\right\rangle \neq 0
$$

This results in a non-Hermitian structure of the Bloch effective Hamiltonian. For the sake of simplicity, we preferred to use Hermitian structure. One possible solution refers to an $\mathrm{S}^{-1 / 2}$ transformation of the projections, and is known as the des Cloizeaux [7] effective Hamiltonian definition. This solution symmetrically distorts projections ; previous work [3] on Heisenberg Hamiltonians for a half-filled band with $2 \mathrm{e}^{-} / 2 \mathrm{AO}$ per centre has shown that this distortion may result in important deviations from the expected structure and difficulties in the transfer of effective operators to larger systems. A better solution consists of a Schmidttype orthogonalization keeping the lowest projected eigenvector unchanged, and orthogonalizing the second one (of higher energy) to the first one. This solution has been applied for ${ }^{1} \Sigma_{\mathrm{g}}^{+}$and ${ }^{3} \Sigma_{\mathrm{u}}^{+}$symmetries.

The ratios of $\left|x_{\mathrm{a}} \bar{x}_{\mathrm{b}}\right|\left(=\left|y_{\mathrm{a}} \bar{y}_{\mathrm{b}}\right|\right)$ over $\left|z_{\mathrm{a}} \bar{z}_{\mathrm{b}}\right|$ projected components are given in table III, other pro- jected eigenvectors on the model space being trivially obtained by symmetry. In this table, the first state of each symmetry corresponds to the lowest in energy. The changes of the order of magnitude (for ${ }^{1} \Sigma_{\mathrm{g}}^{+}$states) and of the signs (for ${ }^{3} \Sigma_{u}^{+}$states), between the two states of each symmetry, occurring between 3.3 and 4.5 a.u., both correspond to avoided crossings. For 3.3 a.u., the two ratios smaller than 1.0 found for ${ }^{1} \Sigma_{\mathrm{g}}^{+}$states do not mean that the states are both $" z^{2}$-dominant "states, since the $[x x]$-component has to be counted twice ( $|x x|$ and $|y y|$ determinants). The first ${ }^{1} \Sigma_{g}^{+}$state is a $[|x x|,|y y|]$-dominant state. The corresponding effective Hamiltonians are given in table IV, where the energy of the two separated atoms (5.18 a.u.) has been taken as zero of energy.

\subsection{COMPARISON BETWEEN THE PERTURBATIVELY} DERIVED AND NUMERICALLY OBTAINED EFFECTIVE HAMILTONIANS. - For the diagonal $R$ "repulsive » matrix elements one should notice (cf. $S_{z}=1$, $[x x, y y, z z]$ symmetry) that $R_{z z}\left(=R_{i}^{\prime}\right)$ is the most repulsive, as expected. The large off-diagonal matrix elements correspond to the effective exchanges $g$. Since the overlap (and hopping integrals) are of larger amplitude in the $\sigma$-system than in the $\pi$ ones, one should have

$$
g_{\sigma \sigma}\left(g_{1}^{\prime}\right)>\left|g_{\sigma \pi}\right|\left(\left|g_{3}\right|\right)>g_{\pi \pi}\left(g_{1}\right) \simeq g_{\pi \pi^{\prime}}\left(g_{2}\right) .
$$

The numerical values actually show the large $g_{\sigma \sigma}$ value ( 0.115 a.u.) at $r=3$ a.u., but the three other integrals are of the same order of magnitude $(-0.03$ to 0.02 a.u.). One should not forget that our third-order perturbative development did not involve hybridized configurations, and deviations are logically expected. Nevertheless the numerical effective Hamiltonian keeps the predicted structure.

Table III. - Ratio of the projected components of $\left|x_{\mathrm{a}} \bar{x}_{\mathrm{b}}\right|$ over $\left|z_{\mathrm{a}} \bar{z}_{\mathrm{b}}\right|$ for the states in the $[x x, y y, z z]-$ symmetry, appearing necessary for the construction of the corresponding $H^{\text {eff }}$.

\begin{tabular}{lrrrrr}
\hline States & \multicolumn{5}{c}{$R$ (a.u.) } \\
& \multicolumn{1}{c}{2.7} & \multicolumn{1}{c}{3.0} & \multicolumn{1}{c}{3.3} & \multicolumn{1}{c}{6.0} \\
\hline${ }^{1} \Sigma_{\mathrm{g}}^{+}$ & 6.32833 & 2.56768 & 0.87945 & 0.18740 & 0.26010 \\
${ }^{1} \Sigma_{\mathrm{g}}^{+}$ & -0.07901 & -0.19473 & -0.56854 & -2.66809 & -1.92231 \\
${ }^{3} \Sigma_{\mathrm{u}}^{+}$ & -13.02096 & -9.58453 & -10.43056 & 28.95102 & 8.68067 \\
${ }^{3} \Sigma_{\mathrm{u}}^{+}$ & 0.03840 & 0.05217 & 0.04794 & -0.01727 & -0.05760 \\
\hline
\end{tabular}


Table IV. - «Exact» numerical $H^{\text {eff }}$ matrix elements at various $B$ - $B$ distances. Deviations from the theoretical third order structure are clearly outlined by a direct comparison to the second column.

\begin{tabular}{|c|c|c|c|c|c|c|}
\hline \multirow{2}{*}{$\begin{array}{c}\text { Space } \\
\text { symmetry }\end{array}$} & \multirow{2}{*}{$\begin{array}{l}\text { Third order } \\
\text { corrected } \\
H^{\text {eff }} \text { matrix } \\
\text { elements }\end{array}$} & \multicolumn{5}{|c|}{$R$ (a.u.) } \\
\hline & & 2.7 & 3.0 & 3.3 & 4.5 & 6.0 \\
\hline$\Sigma^{+}, \Delta$ & $\begin{array}{l}R_{1} \\
R_{1}^{\prime} \\
R_{1}-g_{1} \\
R_{1}^{\prime}-g_{1}^{\prime} \\
g_{1} \\
g_{1}^{\prime} \\
h \\
h^{\prime} \\
\varepsilon_{1} \\
\varepsilon_{1}^{\prime} \\
h+\varepsilon_{1} \\
h^{\prime}+\varepsilon_{1}^{\prime}\end{array}$ & $\begin{array}{r}-0.02880 \\
0.24224 \\
-0.04500 \\
0.12850 \\
0.01620 \\
0.11374 \\
0.00883 \\
0.01010 \\
-0.00076 \\
-0.00780 \\
0.00807 \\
0.00230\end{array}$ & $\begin{array}{r}-0.04350 \\
0.18655 \\
-0.06340 \\
0.07155 \\
0.01990 \\
0.11500 \\
0.00750 \\
0.01167 \\
0.00010 \\
-0.00922 \\
0.00760 \\
0.00245\end{array}$ & $\begin{array}{r}-0.03301 \\
0.15526 \\
-0.05634 \\
0.04352 \\
0.02333 \\
0.11174 \\
0.00606 \\
0.00877 \\
0.00099 \\
-0.00713 \\
0.00705 \\
0.00165\end{array}$ & $\begin{array}{r}-0.00327 \\
0.06182 \\
-0.01703 \\
0.00580 \\
0.01376 \\
0.05603 \\
0.00104 \\
-0.00111 \\
0.00042 \\
-0.00159 \\
0.00145 \\
-0.00270\end{array}$ & $\begin{array}{r}0.00790 \\
0.00650 \\
-0.00991 \\
-0.00703 \\
0.00201 \\
0.01353 \\
-0.00030 \\
-0.00085 \\
0.00009 \\
-0.00086 \\
-0.00021 \\
-0.00171\end{array}$ \\
\hline$\Sigma^{-}, \Delta$ & $\begin{array}{l}R_{2}-g_{2} \\
R_{2}-\bar{g}_{2} \\
\tilde{g}_{2} \equiv g_{2} \\
h+\tilde{g}_{2} \\
\varepsilon_{2} \\
h+\varepsilon_{2}\end{array}$ & $\begin{array}{r}-0.05169 \\
-0.06552 \\
0.02927 \\
0.02789 \\
0.01383 \\
-0.00138\end{array}$ & $\begin{array}{r}-0.06955 \\
-0.08243 \\
0.03288 \\
0.03143 \\
0.01288 \\
-0.00145\end{array}$ & $\begin{array}{r}-0.06323 \\
-0.07376 \\
0.03484 \\
0.03469 \\
0.01053 \\
-0.00015\end{array}$ & $\begin{array}{r}-0.02208 \\
-0.02340 \\
0.01550 \\
0.01009 \\
0.00132 \\
0.00360\end{array}$ & $\begin{array}{r}-0.01005 \\
-0.01030 \\
0.00235 \\
0.00270 \\
0.00025 \\
0.00035\end{array}$ \\
\hline$\Pi$ & $\begin{array}{l}R_{3}-g_{3} \\
R_{3}-\bar{g}_{3} \\
\tilde{g}_{3} \\
h^{\prime}+\overline{\bar{g}}_{3} \\
\varepsilon_{3} \\
h^{\prime}+\varepsilon_{3}\end{array}$ & $\begin{array}{r}-0.02277 \\
-0.04019 \\
-0.00980 \\
-0.00853 \\
0.01742 \\
0.00126\end{array}$ & $\begin{array}{r}-0.04314 \\
-0.05787 \\
-0.03188 \\
-0.03155 \\
0.01472 \\
0.00033\end{array}$ & $\begin{array}{r}-0.03823 \\
-0.05989 \\
-0.04759 \\
-0.03955 \\
0.02167 \\
0.00803\end{array}$ & $\begin{array}{r}-0.02032 \\
-0.02879 \\
-0.02856 \\
-0.02899 \\
0.00847 \\
-0.00043\end{array}$ & $\begin{array}{r}-0.01170 \\
-0.01335 \\
-0.00555 \\
-0.00715 \\
0.00165 \\
-0.00160\end{array}$ \\
\hline
\end{tabular}

The effective Hamiltonians have been calculated for five interatomic distances. All matrix elements $R, g, \varepsilon$ decrease exponentially with the interatomic distance, since they involve interatomic overlap distributions. The quadrupole-quadrupole interactions $h$ decrease as $r^{-8}$. Analytic fittings of the matrix elements are easy and may be used for geometry optimizations in larger clusters.

\section{Transferability to the linear $B_{3}$ system.}

Transferability of the effective Hamiltonian has been tested on the simplest larger problem, namely the $B_{3}$ linear system keeping $D_{\infty h}$ symmetry.

4.1 Qualitative Considerations. - Close to the equilibrium geometry, the lowest states of $\mathrm{B}_{2}$ are, in increasing order : ${ }^{3} \Sigma_{g}^{-}$and ${ }^{1} \Delta_{g}([x y]$-symmetry $\longrightarrow)$ and ${ }^{3} \Pi_{\mathrm{u}}\left([x z]\right.$-symmetry : $\left.{ }^{-}\right)$. Starting from two B interactions to form the linear $B_{3}$ molecule, we may predict to find, as lowest states for $B_{3}$, a structure resulting from the junction of two such $[x y]$-structures $\zeta,-$ or one $[x y]$ - and one $[x z]$-structure : $\longleftarrow$, Moreover, as also outlined in the introduction, the distribution corresponding to all spins parallel, i.e. a quartet symmetry in that case, should be low in energy.

4.2 AB INITIO MO-CI CAlCulations. - Accurate MO-CI calculations have been performed on $B_{3}$. The symmetry of the $B_{3}$ ground state was investigated first. Keeping in mind that $d$-functions were of fundamental importance in the determination of the right symmetry for the ground state of $B_{2}$, we used the same triple- $\zeta_{s}$, double- $\zeta_{p}$ and single $\zeta_{d}$ valence basis set as for $B_{2}$. According to the CIPSI algorithm, a great part of the valence correlation energy has been treated variationally and the remaining part perturbatively. Figure 5 shows the three lowest states close to the equilibrium internuclear distance which is found of the same order as that of $B_{2}$. The ${ }^{2} \Pi_{u}$ state is the ground state. The quartets ${ }^{4} \Pi_{\mathrm{g}}$ and ${ }^{4} \Sigma_{\mathrm{u}}^{-}$, suggested by our qualitative considerations, are only about 0.006 a.u. above the 


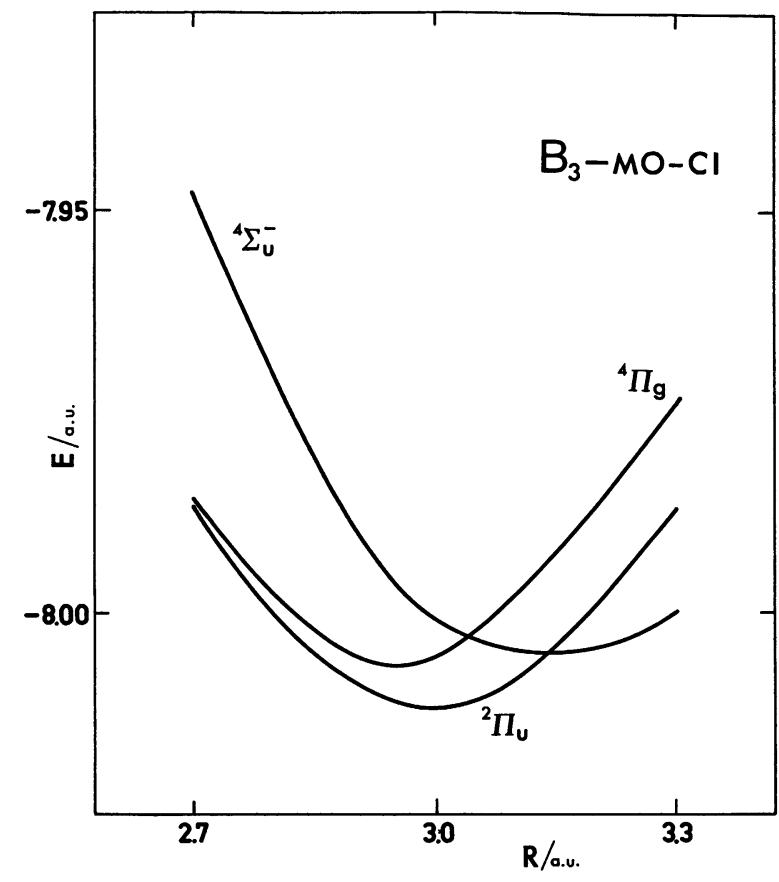

Fig. 5. - MO-CI potential curves for the three lowest states of $B_{3}$.

ground state, the ${ }^{4} \Pi_{\mathrm{g}}$ state being slightly more stable than the ${ }^{4} \Sigma_{u}^{-}$state.

In a second step, in order to lighten the calculations, we eliminated the d-functions from the basis set. Figures 6a and 7a show the lowest states for the three subsymmetries. These three subsymmetries are denoted here: $[x x x]$ (which also includes $|x y y|$ and $|x z z|$ determinants and the degenerate ones), $[z z z]$ (with also $|x x z|$ and $y y z$ determinants) and $[x y z]$. The $\mathrm{u}$ and $\mathrm{g}$ characters are then assigned appropriatly to each MO in MOCI calculations while $a, b$ and $c$ subscripts characterize the atoms to each A.O. in the V.B. formalism. The three lowest states are still found to be : ${ }^{2} \Pi_{\mathrm{u}},{ }^{4} \Pi_{\mathrm{g}}$ and ${ }^{4} \Sigma_{\mathrm{u}}^{-}$in the same order.

4.3 COMPARISON BETWEEN $A B$ INITIO AND EFFECTIVE HAMILTONIAN RESULTS FOR $\mathrm{B}_{3}$. - Malrieu $e t$ al. [5] discussed in detail the problem of the orthogonality between A.O.'s. The orthogonalized A.O.'s. for a two-atom system and those for a three-atom system are not the same. We must keep in mind that this could affect the transferability to some extent. Their calculations on alkali metals also have shown that, as expected, three-body increments $\delta g_{\mathrm{ab}}$ (c) are small, at least in linear chains.

The $B_{3}$ effective Hamiltonian splits into three blocks of dimensions 18, 21 and 21 for the $[x y z],[x x x]$ and [zzz] pseudo-symmetries respectively. The long-distance (A-C)-interactions have been checked to have negligible effects. The ground state is correctly predicted to be of ${ }^{2} \Pi_{\mathrm{u}}$ symmetry by our model, and the

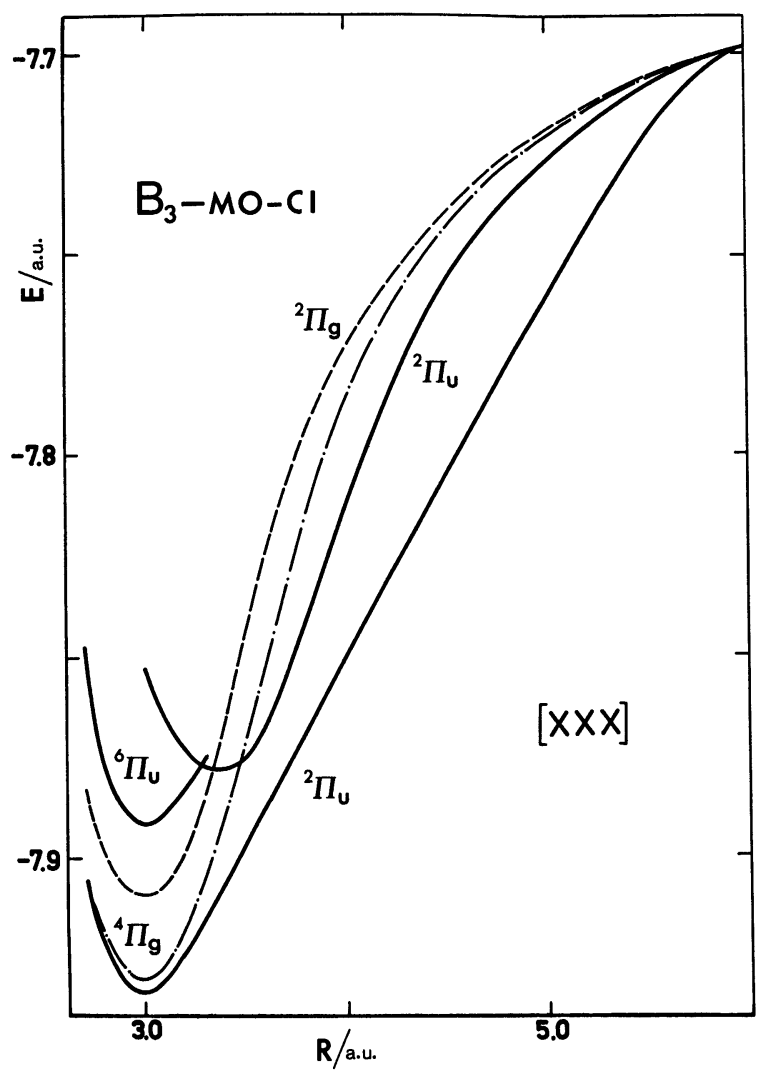

Fig. 6a. - MO-CI $[x x x]$-symmetry lowest potential curves of $\mathrm{B}_{3}$.

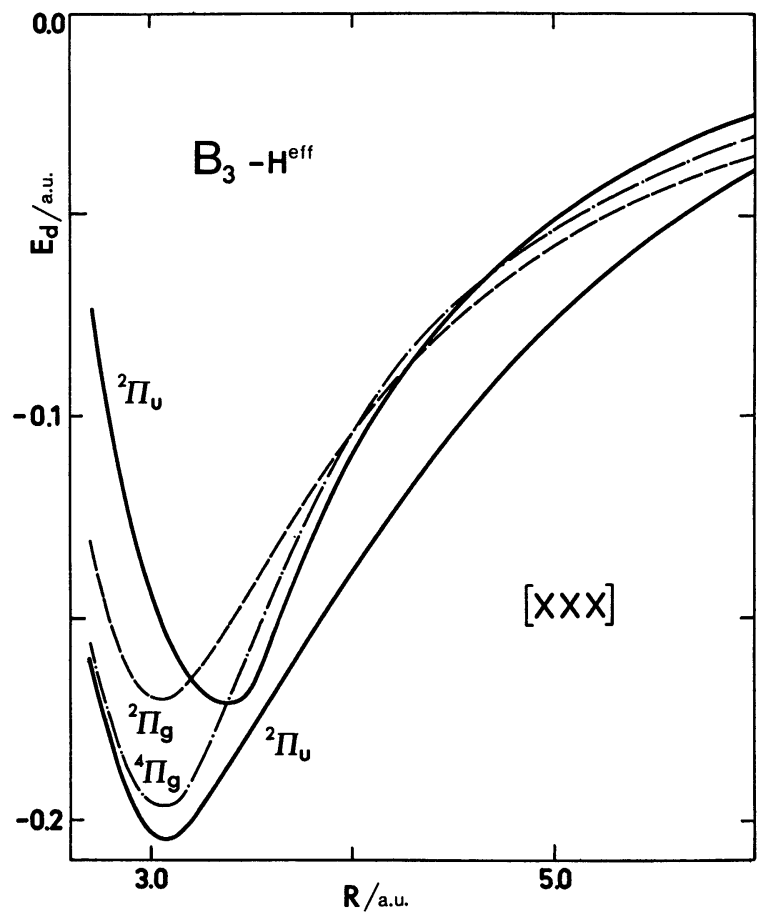

Fig. 6 b. $-H^{\text {eff }}[x x x]$-symmetry lowest potential curves of $\mathrm{B}_{3}$. 


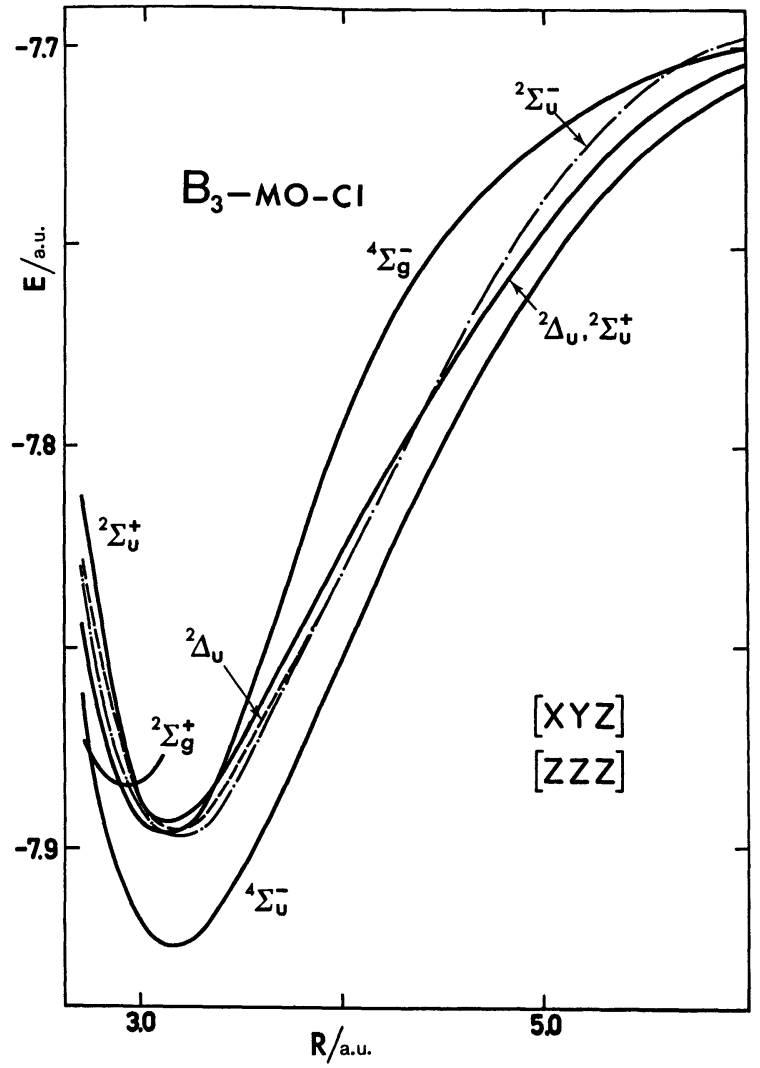

Fig. 7a. - MO-CI $[x y z]$ - and $[z z z]$-symmetry lowest potential curves of $B_{3}$.

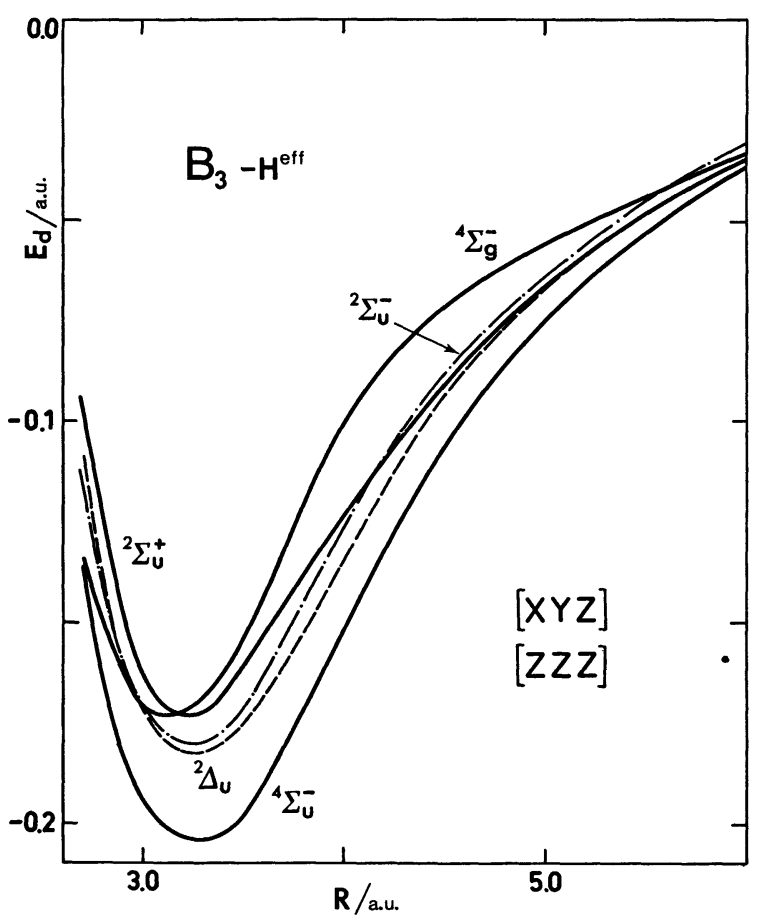

Fig. 7b. $-H^{\mathrm{eff}}[x y z]$ - and $[z z z]$-symmetry lowest potential curves of $\mathrm{B}_{3}$. equilibrium distance is correct (3.08 a.u. to be compared to 3.01 in the $a b$ initio calculations). From a V.B. point of view it may be analysed as a resonance between

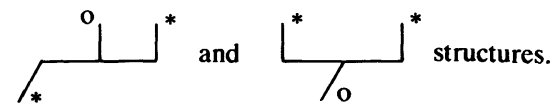

The two upper states are very close in energy ; they are quartets of ${ }^{4} \Pi_{\mathrm{g}}$ and ${ }^{4} \Sigma_{\mathrm{u}}^{-}$symmetries. The best MO-CI calculation predicts them at $T_{\mathrm{e}}=0.14 \mathrm{eV}$ and $0.19 \mathrm{eV}$ above the ground state minimum, while the effective Hamiltonian predicts 0.22 and $0.03 \mathrm{eV}$ respectively: this inversion corresponds to an error of $\simeq 0.2 \mathrm{eV}$, which is not too bad. Deleting the d A.O.'s in the MOCI calculation leads to $T_{\mathrm{e}}=0.11$ and $0.24 \mathrm{eV}$ respectively for ${ }^{4} \Pi_{\mathrm{g}}$ and ${ }^{4} \Sigma_{\mathrm{u}}$. The important feature is the differential effect on the interatomic distances (cf. Figs. 6-7 and Table V) which are correctly reproduced by the effective Hamiltonian. The V.B. content of these wave functions may be pictured as

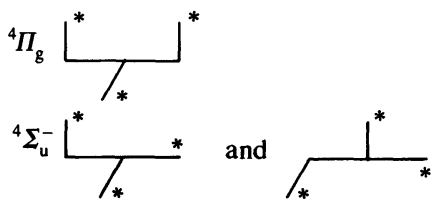

A direct comparison between the potential curves of upper states may be obtained by comparing figures $6 a$ and $6 \mathrm{~b}$ for the states of $\Pi$ and $\Phi$ symmetries, and figures $7 \mathrm{a}$ and $7 \mathrm{~b}$ for the states of $\Sigma^{-}, \Delta$ and $\Sigma^{+}$ symmetries.

The results are also summarized in table $\mathrm{V}$. There is a significant gap $(\simeq 0.5 \mathrm{eV})$ between the three lowest states and a group of five states which lie in a narrow energy range of $0.4 \mathrm{eV}$. These states are of ${ }^{2} \Pi_{\mathrm{g}},{ }^{2} \Sigma_{\mathrm{u}}^{-},{ }^{4} \Sigma_{\mathrm{g}}^{-},{ }^{2} \Delta_{\mathrm{u}}$ and ${ }^{2} \Sigma_{\mathrm{u}}^{+}$character, in order of increasing energy in the MO-CI calculation. The effective Hamiltonian only permutes the position of the ${ }^{2} \Pi_{\mathrm{g}}$ and ${ }^{2} \Delta_{\mathrm{u}}$ states in this group. The largest equilibrium distances concern the ${ }^{2} \Sigma_{\mathrm{u}}^{-}$and ${ }^{2} \Delta_{\mathrm{u}}$ states in both approaches.

The two next states are ${ }^{6} \Pi_{\mathrm{u}}$ and ${ }^{2} \Sigma_{\mathrm{g}}^{+}$in the MO-CI calculation. The former cannot be reached from a three-open-shell model space and the second one is essentially hybridized; from that region important discrepancies begin to appear between the MO-CI and effective Hamiltonian spectra.

\section{Conclusion.}

The major conceptual interest of the present derivation concerns the logical possibility and cost of an extension of the Heisenberg Hamiltonian to a non half-filled band problem. The basic philosophy is again the choice of a model space spanned by the neutral V.B. determinants, 
Table V. - Lowest spectroscopic states of $\mathrm{B}_{3}$. The values in parentheses are relative to the largest basis set.

\begin{tabular}{lcccccc}
\hline \multirow{2}{*}{ States } & \multicolumn{2}{c}{$T_{\mathrm{e}}(\mathrm{eV})$} & \multicolumn{2}{c}{$\Delta E(\mathrm{vert})(\mathrm{eV})$} & \multicolumn{2}{c}{$r_{\mathrm{e}}(\mathrm{a} . \mathrm{u})}$. \\
& MOCI & $H^{\text {eff }}$ & MOCI & $H^{\text {eff }}$ & MOCI & $H^{\text {eff }}$ \\
\hline${ }^{2} \Pi_{\mathrm{u}}$ & 1.52 & 0.90 & 2.20 & 1.41 & 3.34 & 3.40 \\
${ }^{2} \Sigma_{\mathrm{g}}^{+}$ & 1.36 & - & 1.39 & - & 2.94 & - \\
${ }^{6} \Pi_{\mathrm{u}}$ & 1,14 & - & 1.14 & - & 3.00 & - \\
${ }^{2} \Sigma_{\mathrm{u}}^{+}$ & 1.09 & 0.87 & 1.25 & 1.03 & 3.12 & 3.24 \\
${ }^{2} \Delta_{\mathrm{u}}$ & 1.06 & 0.60 & 1.22 & 0.82 & 3.20 & 3.26 \\
${ }^{4} \Sigma_{\mathrm{g}}^{-}$ & 1.03 & 0.87 & 1.09 & 0.90 & 3.10 & 3.12 \\
${ }^{2} \Sigma_{\mathrm{u}}^{-}$ & 1.01 & 0.65 & 1.20 & 0.79 & 3.20 & 3.26 \\
${ }^{2} \Pi_{\mathrm{g}}$ & 0.68 & 0.92 & 0.68 & 0.92 & 3.00 & 3.06 \\
${ }^{4} \Sigma_{\mathrm{u}}^{-}$ & 0.24 & 0.03 & 0.41 & 0.14 & 3.15 & 3.30 \\
& $(0.19)$ & & $(0.30)$ & & $(3.14)$ & \\
${ }^{4} \Pi_{\mathrm{g}}$ & 0.11 & 0.22 & 0.11 & 0.24 & 3.00 & 3.06 \\
${ }^{2} \Pi_{\mathrm{u}}$ & $(0.14)$ & $0.19)$ & & $(2.95)$ & 3.00 & 3.08 \\
& 0 & 0 & 0 & & $(3.00)$ & \\
\hline
\end{tabular}

and the use of quasi degenerate perturbation theory or spectral definition of the effective Hamiltonian. The use of the perturbative expansion has the advantage of putting in place the logical structure of $H^{\text {eff }}$, showing which are the leading terms and their physical origin. The use of the spectral definition of $H^{\text {eff }}$ permits avoidance of divergence problems of the perturbative expansion and introduces high-quality (infinite order) information.

The effective operators involve, beside the classical Heisenberg type spin exchange operators between A.O.'s of the same subsystem :

- spin-exchange operators between A.O.'s of two orthogonal subsystems ;

- angular momenta-exchange operators ;

- spin- and angular momenta-exchange operators ; the two last types implying necessarily four A.O.'s belonging to two orthogonal subsystems.

The generalization of the Heisenberg effective Hamiltonian approach to $(\mathrm{p} / n)$-filled bands is thus in principle possible although some supplementary difficulties may arise from the existence of $p$ electrons per atom. This approach may be useful for the study of the $d^{n}$ systems of clusters of transition metals.

Concerning the precise case studied here, the main problem concerned of course the restriction of our model to a single band through the choice of a model space spanned by products of atomic $\mathrm{s}^{2} \mathrm{p}$ configurations. The hybridization (i.e. the involment of $\mathrm{sp}^{2}$ atomic configurations) should play a very important role, and this is clear from the existence of a low lying quintet state ${ }^{5} \Sigma_{u}^{-}$of $B_{2}$, which cannot enter our model. The fact that we used the exact eigenenergies of $B_{2}$ for the spectral definition of $H^{\text {eff }}$ has taken implicitly into account most of the hybridization mixing. One might have defined the target space $S$ as spanned by the eigenvectors of $H$ having the largest components on the model space $\mathrm{S}_{0}$, some of them being higher in energy. Attempts along this line failed to give reliable results except for a few almost non-hybridized states (the ground state was correctly predicted to be ${ }^{2} \Pi_{u}$ ). The success of our model for $B_{3}$ is certainly linked to the fact that the strongly hybridized states go to higher energies on linear chains at least, since the lowest essentially hybrid state ${ }^{6} \Pi_{u}$ appears only in $9^{\text {th }}$ position in the MO-CI calculation.

Such success would be unlikely for bi- and tridimensional clusters. We intend to apply our effective Hamiltonians to the prediction of the magnetic character of linear $\mathrm{B}_{n}$ chains.

It is clear that the main further step in the construction of effective Hamiltonians spanned by all neutral V.B. structures would consist in explicitly treating the hybridization, i.e. several bands, spanning the model space by all the possible products of neutral ground and excited valence atomic configurations. This strategy, now under study, faces three types of problem;

- the large size of the model space makes the $a b$ initio extraction difficult; the use of the recently proposed intermediate Hamiltonians [23] may solve some intruder state problems, necessarily occurring in such situations ;

- effective integrals will be numerous, and their handling in larger problems may become tedious. Some analytic fittings of effective integrals (in terms of overlap for instance) may become necessary ;

- the number of neutral V.B. determinants for a moderate-size cluster or molecule will be large and the diagonalization of the generalized Heisenberg Hamiltonian matrix may become rather expensive. Sanchez et al. [24] recently proposed a procedure to truncate 
and dress these large matrices insuring numerical accuracy and size-consistence. Despite these practical difficulties it is worthwile knowing that the effect of electronic delocalization may be treated through «effective » interatomic exchange operators acting on neutral V.B. structures only, and that this philosophy usually limited to half-filled bands, is completely general.

\section{References}

[1] Herring, C., in Magnetism, edited by C. T. Rado and M. Suhl (Academic Press, New York) 1966, Vol. II B.

[2] Anderson, P. W., Phys. Rev. 115 (1959) 1 ; in Solid State Physics, edited by H. Ehrenreich, F. Seitz, and D. Turnbull (Academic Press, New York) 1963, Vol. 14, p. 99 ;

Brandow, B. H., Adv. Phys. 26 (1977) 651.

[3] Maynau, D., Garcia-BaCh, M. A. and Malrieu, J. P., J. Physique 47 (1986) 207.

[4] Malrieu, J. P. and Maynau, D., J. Am. Chem. Soc. 104 (1982) 3021 ;

Maynau, D. and Malrieu, J. P., J. Am. Chem. Soc. 104 (1982) 3029.

[5] Malrieu, J. P., Maynau, D. and Daudey, J. P., Phys. Rev. B 30 (1984) 1817.

[6] Bloch, C., Nucl. Phys. 6 (1958) 329.

[7] Des Cloizeaux, J., Nucl. Phys. 20 (1960) 321.

[8] Van Vleck, J. H., Phys. Rev. 33 (1929) 467 ;

Des Cloizeaux, J., Nucl. Phys. 20 (1960) 321 ;

Brandow, B. H., Rev. Moc'. Phys. 39 (1967) 771 ;

Kirtman, B., J. Chem. Phys. 49 (1968) 3890 ;

Certain, P. R. and Hirschfelder, O., ibid. 52 (1970) 5977 ;

Hose, G. and Kaldor, U., J. Phys. B 12 (1979) 3827 ;

Shavitt, I. and Redmon, L. T., J. Chem. Phys. 73 (1980) 5711.

[9] Durand, Ph., Phys. Rev. A 28 (1983) 3184.

[10] Padgett, A. A. and Griffing, V., J. Chem. Phys. 30 (1959) 1286.

[11] Bender, C. F. and Davidson, E. R., J. Chem. Phys. 46 (1967) 3313.

[12] Douglas, A. F. and Herzberg, G., Can. J. Res. A 18 (1940) 165.
[13] Bader, R. F. W., Henneker, W. H. and Cade, P. E., J. Chem. Phys. 46 (1967) 3341.

[14] Dupuis, M. and Liu, B., J. Chem. Phys. 68 (1978) 2902.

[15] Becke, A. D., J. Chem. Phys. 76 (1982) 6037 ;

DunlaP, B. I., Ibid 78 (1983) 4787 ;

BeCKe, A. D., Ibid 78 (1983) 4787.[16] DE B. DARWENT, B., Bond dissociation energies in simple molecules, NSRDS-NBS 31 (Natl. Bur. Stand., Washington D.C.) 1970 ;

Huber, K. P., American Institute of Physics Handbook, edited by D. C. Gray (McGraw-Hill, New York) 1972, Sec. $7 \mathrm{~g}$;

Huber, K. P. and Herzberg, G., Molecular spectra and molecular structure. Constants of diatomic molecules (Van Nostrand Reinhold Cy, New York) 1979, p. 64.

[17] Handbook of Chemistry and Physics, Ed. by R. C. Weast, $64^{\text {th }}$ ed. (C.R.C. Press Inc.) 1983-1984.

[18] DAudeY, J. P., private communication.

[19] Dupuis, M., RYs, J. and KING, H. F., J. Chem. P: hys. 65 (1976) 111 ;

Dupuis, M. and KInG, H. F., Int. Quant. Chem. 11 (1977) 613.

[20] Calculs atomiques et moléculaires ab initio : Technical report, U.A. 505, Lab. Phys. Quant. Univ. Paul Sabatier, Toulouse, 1981.

[21] Huron, B., Malrieu, J. P. and Rancurel, P., J. Chem. Phys. 58 (1973) 5745.

[22] Epstein, P. S., Phys. Rev. 28 (1926) 695 ;

NeSBet, R. K., Proc. R. Soc. London A 230 (1955) 312, 322.

[23] Malrieu, J. P., Durand, Ph. and Daudey, J. P., J. Physics A Math. Gen. 18 (1985) 809.

[24] Sanchez-Marin, J., Malrieu, J. P. and Maynau, D., Int. J. Quant. Chem. in press. 